\title{
Transverse lattice approach to light-front Hamiltonian QCD
}

\author{
S. Dalley* \\ Theory Division, CERN, CH-1211 Geneva 23, Switzerland
}

B. van de Sande

Institut Für Theoretische Physik III, Staudstraße 7, D-91058 Erlangen, Germany

(Received 11 August 1998; published 11 February 1999)

\begin{abstract}
We describe a nonperturbative procedure for solving from first principles the light-front Hamiltonian problem of $\mathrm{SU}(N)$ pure gauge theory in $D$ spacetime dimensions $(D \geqslant 3)$, based on enforcing Lorentz covariance of observables. A transverse lattice regulator and color-dielectric link fields are employed, together with an associated effective potential. We argue that the light-front vacuum is necessarily trivial for large enough lattice spacing and clarify why this leads to an Eguchi-Kawai dimensional reduction of observables to $1+1$ dimensions in the $N \rightarrow \infty$ limit. The procedure is then tested by explicit calculations for $(2+1)$-dimensional $\mathrm{SU}(\infty)$ gauge theory, within a first approximation to the lattice effective potential. We identify a scaling trajectory which produces Lorentz-covariant behavior for the lightest glueballs. The predicted masses, in units of the measured string tension, are in agreement with recent results from conventional Euclidean lattice path integral simulations. We are able to go further, studying the longitudinal and transverse structure of glueballs using their light-front wave functions. Finally, we briefly discuss the extension of these calculations to $3+1$ dimensions. [S0556-2821(99)05004-3]
\end{abstract}

PACS number(s): 11.15.Tk, 12.38.Aw

\section{INTRODUCTION}

Light-front Hamiltonian quantization is a very promising approach to the nonperturbative calculation and understanding of hadronic substructure, as a function of the quark and gluonic degrees of freedom. This is both of intrinsic interest and important for the proper identification of new physics beyond the standard model. Recently, we have developed an idea [1], originally suggested by Bardeen and Pearson many years ago [2,3], for formulating the light-front Hamiltonian of QCD on a transverse lattice. The continuum limit is taken in time and one space direction, while the remaining transverse degrees of freedom are represented as color-dielectric link variables [4] in a gauge-invariant way on the transverse lattice. Since light-front wave functions carry nonperturbative, coherent information about hadronic bound and scattering states in a general Lorentz frame, a solution to this problem would open up vast amounts of existing and future experimental results to theoretical scrutiny [5]. We believe that the menagerie of light-front Hamiltonian, transverse lattice, and effective color-dielectric fields is a very potent combination in the analysis of the strong interactions at intermediate scales. Each contributes to the solution of long-standing technical problems associated with the others. In addition, profound simplifications take place when one considers only the leading order of the $1 / N$ expansion [6] in the number of colors, $N$. In this paper, we describe in detail how nonperturbative first-principles calculations may be performed for pure gauge theory, in $2+1$ and $3+1$ dimensions, by imposing Lorentz covariance on physical observables. The procedure is then tested with explicit numerical and analytic computa-

\footnotetext{
*On leave from DAMTP, University of Cambridge, Cambridge CB3 0DS, England.
}

tions for $2+1$ dimensions at large $N$. Our glueball spectrum compares successfully with recent "state-of-the-art" results from conventional Euclidean lattice simulations. Having also the light-front wave functions, we are able to go further, studying the longitudinal and transverse internal structure of these glueballs.

The choice of dynamical variables and regulator in canonical light-front quantization is crucial to a practical solution. The well-known Lorentz boost invariance of light-front wave functions comes with a price, because, for example, the light-front quantization surface is not rotationally invariant; typically, the regulators chosen will break rotational invariance. An effective potential must be introduced to restore full Lorentz invariance. This effective potential plays another important role to encode what is usually, that is, in equaltime quantization, termed "vacuum structure." One of the attractive features of light-front quantization is that, in the true ground state of a theory, the light-front vacuum is trivial-there can be no pair creation-except possibly for some massless gauge modes of zero momentum. But in the true ground state, much of the complication usually attributed to the vacuum has only been shifted into effective interactions appearing in the Hamiltonian.

Typically, a light-front Hamiltonian will consist of a "bare" part $H_{B}$ derived from the classical action and the effective potential consisting of counterterms $H_{C}$. The role of the latter is to enforce the fundamental (unbroken) symmetries required of the theory, which are broken by the choice of regulators. For practical purposes, one must decide on some physical grounds which subset of all possible operators one is going to consider in $H_{C}$, a subset which will then be systematically enlarged as greater accuracy is demanded. The method we will use in our work, to fix the couplings to this subset of operators, is to solve the entire 
theory as a function of the couplings, which are then tuned by demanding unbroken symmetries of calculated observables. This straightforward approach will allow direct calculations involving typical hadronic scales, intermediate between the asymptotically free region and nuclear scales. Naively, one might think that the approach is computationally unviable, but this is not the case if one uses Hamiltonian methods. There already exists at least one successful application of this approach to fixing counterterms. Parity, which is not a manifest symmetry of the light front, is typically broken by regulators. Burkardt [7] has shown how, in the case of the Yukawa model in $1+1$ dimensions, a simple counterterm coupling can be adjusted to restore parity invariance in a suitable observable. The general problem in higher dimensions involves the far more complicated task of ensuring continuous Lorentz symmetries also. This will form the main part of our job in solving pure gauge theory.

To realize the above scenario, we apply light-front quantization to a set of massive effective degrees of freedom on the transverse lattice-color-dielectric link variables-which efficiently organize a gauge-invariant Hamiltonian in the true vacuum. These variables are supposed to represent an average over the high-frequency transverse gluon degrees of freedom. The fact that (lattice) gauge invariance is not broken helps to restrict the number of counterterms needed in $H_{C}$ and leads to a simple understanding of confinement. The dielectric variables will provide the required physical basis for systematic approximation to the low-energy effective potential. Moreover, they are flexible enough that in the lightfront Hamiltonian formulation one can solve for physical observables, with reasonable computational effort, as a function of the couplings in the effective potential. This formulation therefore satisfies the requirements necessary for our approach. In the first half of this paper, we construct in detail the framework for making calculations in pure gauge theory at large $N$. This framework is developed in $3+1$ dimensions, but can also be applied in $2+1$ dimensions with some trivial modifications.

As a quantitative test in a highly relativistic, strongly interacting system, we then perform detailed calculations in 2 +1 dimensions. This choice as a testing ground is motivated by a number of reasons. A more reliable computation can be performed in $2+1$ dimensions than $3+1$ dimensions. Teper [8] has recently completed extensive and precise conventional Euclidean lattice Monte Carlo (ELMC), simulations in three dimensions for various $N$. The resulting spectra may be extrapolated to large $N$, providing a benchmark for our work. Finally, the physics of Yang-Mills theory in $2+1$ dimensions is remarkably similar that in $3+1$ dimensions.

In our previous work [1] (hereafter referred to as I), we studied the effective potential in $2+1$ dimensions by matching our glueball spectrum to Teper's preliminary data. For a truncated effective potential, we were able to identify a coupling constant trajectory along which mass ratios were approximately constant and agreed reasonably well with the ELMC continuum predictions. This is a perfectly acceptable phenomenological approach, but one would obviously like to avoid fitting to an existing spectrum, whether taken from experiment or another theoretical method. The first- principles calculations we perform in this paper use only general concepts like gauge and Lorentz invariance to fix the effective potential. In this way, we can make independent predictions of all physical quantities. The contents of the present paper are summarized below.

In the next section, we briefly review the color-dielectric formulation of lattice gauge theory and its light-front Hamiltonian limit in order to set the notation. In Sec. III, we carry out the light-front quantization, arguing that in the "colordielectric regime" where the light-front Hamiltonian is quantized, light-front zero modes may be neglected; that is, the light-front vacuum is essentially trivial. We observe that linear confinement is generic to the theory in the colordielectric regime. All our work is performed in the large- $N$ limit because of remarkable simplifications which occur. In Ref. [9] and I it was proved that, in this limit, one effectively solves the light-front Hamiltonian of a $(1+1)$-dimensional gauge theory coupled to $2^{D-2}$ adjoint matter fields, for $D$ space-time dimensions. We clarify in Sec. V the physical role of this Eguchi-Kawai dimensional reduction. Unlike lattice path integral quantization, where in the leading order in $N$ vacuum structure undergoes dimensional reduction to zero dimensions, the leading order in $N$ of the transverse lattice light-front quantization exhibits dimensional reduction of physical observables to $1+1$ dimensions. Section VI outlines procedures for measuring the string tension in the transverse lattice direction via winding modes and the full spatial potential between two heavy sources, extending work of Ref. [10]. In Sec. VII, we introduce states with nonzero transverse momentum. We use the resulting dispersion relation, together with the string tension measurements, to construct a simple algorithm for fixing the effective potential based on Lorentz covariance.

We then turn to a numerical implementation of our procedure for $(2+1)$-dimensional gauge theory (Sec. VIII). Most of the relevant formulas and descriptions of our numerical methods have been given in I; we briefly review them in Sec. VIII A. Section IX presents our results for the scaling behavior, glueball masses and wave functions, the string tensions, and heavy-source potential. Finally, we conclude with a brief discussion of the extension to $3+1$ dimensions of these calculations. A number of the more technical steps of the paper have been relegated to the Appendixes.

\section{COLOR-DIELECTRIC LATTICE GAUGE THEORY}

\section{A. Euclidean lattice}

For the purposes of studying a light-front Hamiltonian limit, Wilson's formulation of lattice gauge theory [11] leads to difficulties due to the nonlinearity of the link variables [12]. We therefore take as our starting point Mack's linearized "color-dielectric" formulation of lattice gauge theory [4]. A similar lattice gauge theory was first introduced by Weingarten [13] as a generator of the spacetime lattice regularization of the Nambu string action.

On a four-dimensional hypercubic lattice of spacing $a$, introduce a complex matrix $M_{\mu}(x) \in G L(N, C)$ for the link attached to site $x=\left\{x^{0}, x^{1}, x^{2}, x^{3}\right\}$ in the direction $x^{\mu}$. The partition function is 


$$
Z=\int \sum_{x, \mu} \sum_{i, j=1}^{N} d M_{\mu, i j}(x) d M_{\mu, i j}^{*}(x) \exp (-S[M]),
$$

with color indices $i, j \in\{1, \ldots, N\}$ and $M_{\mu}(x)=M_{-\mu}^{\dagger}(x$ $+a \hat{\mu})$. Throughout, $\hat{\mu}$ is the unit vector in the $x^{\mu}$ direction and $\widehat{-\mu}=-\hat{\mu}$. For an appropriate choice of action $S$, this theory is invariant under lattice gauge transformations

$$
M_{\mu}(x) \rightarrow U^{\dagger}(x) M_{\mu}(x) U(x+a \hat{\mu}), \quad U \in \mathrm{SU}(N) .
$$

Since we will eventually be interested in an expansion of the action in powers of the field $M$ in the large- $N$ limit, we take $S[M]$ to consist of Wilson loops and their products. Note that this will include Wilson lines which 'backtrack,' such as $\operatorname{Tr}\left\{M_{\mu}(x) M_{\mu}^{\dagger}(x)\right\}$, because $M$ is not necessarily a unitary matrix. (Determinants of $M$ are also gauge invariant, but involve an infinite product of fields when $N=\infty$.) Since we are interested in QCD, $S$ is tuned in such a way that, as the correlation length approaches infinity in direction $x^{\mu}$ (lattice spacing $a \rightarrow 0$ in physical units),

$$
\lim _{a \rightarrow 0} M_{\mu}(x)=1+i a A_{\mu}+O\left(a^{2}\right),
$$

where $A_{\mu}$ is the usual Hermitian gauge potential. Thus $M$ must tend asymptotically to the unitary Wilson link variable [11], and in the isotropic continuum limit, $S$ will be proportional to $\operatorname{Tr}\left\{F_{\mu \nu} F^{\mu \nu}\right\}$. For future reference, it is convenient to introduce lattice versions of the covariant derivative and field strength tensor [4];

$$
\bar{D}_{\mu} M_{\nu}(x) \equiv M_{\mu}(x) M_{\nu}(x+a \bar{\mu})-M_{\nu}(x) M_{\mu}(x+a \hat{\nu}) .
$$

This natural definition reduces to $F_{\mu \nu}$ in the continuum.

More generally, the coefficients of $S$ are to be chosen to obtain scaling; that is, Green's function observables are to be independent of the correlation length(s). We will demonstrate existence ${ }^{1}$ of an approximate scaling trajectory, in the light-front Hamiltonian limit, by demanding Lorentz covariance of bound-state solutions to the theory with $S$ truncated to a finite number of terms. This empirical approach is similar in spirit to the Monte Carlo renormalization group [14]. However, we find it more convenient to directly minimize violations of Lorentz covariance in observables, as a function of couplings in $S$, rather than try to perform explicit "blockspin" transformations.

The Wilson loops up to length 4 are illustrated in Fig. 1. The quadratic loop $\operatorname{Tr}\left\{M_{\mu}(x) M_{\mu}^{\dagger}(x)\right\}$, that is, the "masssquared" term of the $M_{\mu}(x)$ fields, evidently plays a special role. It must obviously have negative (tachyonic) coefficient in the continuum limit (3). If it has a positive coefficient, a "strong-coupling" expansion of the path integral (1) about

\footnotetext{
${ }^{1}$ Uniqueness would follow from the assumption that the continuum limit of QCD is unique, since the light-front Hamiltonian approach requires us to take the continuum limit (3) in two directions and then demand approximate Lorentz invariance.
}

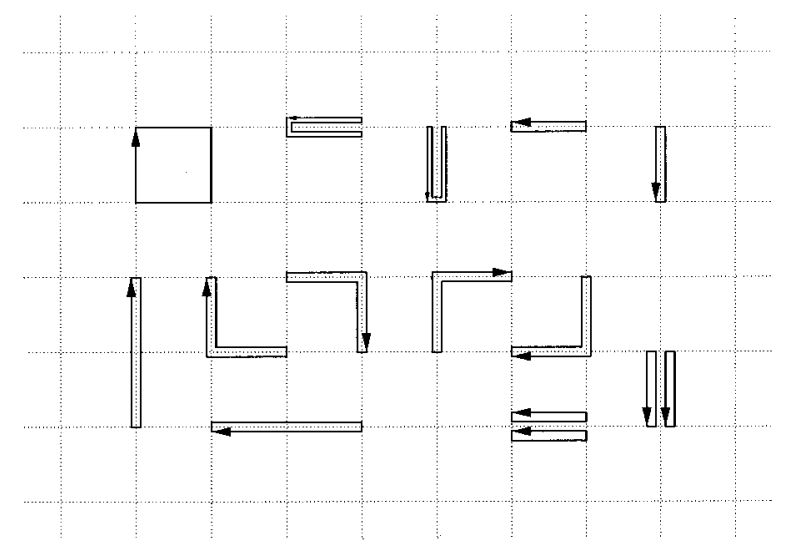

FIG. 1. All Wilson loops and their local products of total length $\leqslant 4$. To those shown above one must add the different orientations of each loop in $D$ spacetime dimensions and reversal of arrows (only the plaquette gives a distinct loop in the latter case.) In the case of products of Wilson loops, we have only shown the case where the loops lie exactly on top of one another (local product).

$M_{\mu}(x)=0$ may be performed [13]. If the scaling trajectory passes through such a region, Mack showed that a colordielectric picture of confinement results $[4,15]$. Accordingly, we shall refer to this as the color-dielectric regime. There is evidence for $\mathrm{SU}(2)$ that a partition function of the form (1), subject to "block-spin" transformations, does have renormalization group trajectories which pass into the colordielectric regime at short enough correlation length [16]. Therefore, the picture one should keep in mind is the following. If one decomposes $M_{\mu}(x)=H U$ into a Hermitian matrix $H$ and a unitary matrix $U$, in the continuum limit $a \rightarrow 0, U$ is identified with Wilson's link variable. $H=H_{0}+H$ gets a vacuum expectation value $(\mathrm{VEV}) H_{0}$, while the fluctuation $\widetilde{H}$ becomes very heavy and decouples. Near the continuum limit, $H_{0}$ appears in the equations of motion like a generalized dielectric constant [4]. In this regime the field $M_{\mu}(x)$ is tachyonic. As the lattice spacing $a$ is increased, a scaling trajectory in $S$ pushes one into a region of positive mass squared for $M_{\mu}(x)$, where $H_{0}$ vanishes and $\widetilde{H}$ is fully dynamical. The mass of $M_{\mu}(x)$ then increases with $a$. We will find further evidence for this in our light-front Hamiltonian calculations later, where it is rather crucial to work in a regime of positive mass squared for $M_{\mu}(x)$.

\section{B. Light-front continuum limit}

It is difficult to perform quantitative calculations with the dielectric lattice gauge theory described above because one must search the multidimensional coupling constant space of $S$ for a scaling trajectory. We will show that these problems can be overcome in the light-front Hamiltonian limit. To employ a light-front Hamiltonian formulation, it is desirable to take the continuum limit in two directions. Thus, in $D$ spacetime dimensions, we will take the lattice spacing to zero in the $x^{0}$ and $x^{D-1}$ directions while keeping the lattice spacing $a$ in the $D-2$ remaining "transverse", directions constant. Throughout, boldface will denote vectors in the 
transverse directions, b, with components $b^{r}$ where $r, s$ $\in\{1, \ldots, D-2\}$. Continuum Lorentz indices are denoted by $\alpha, \beta \in\{0, D-1\}$; thus, we write the inner product of two Lorentz vectors as $a^{\mu} b_{\mu}=a^{\alpha} b_{\alpha}-\mathbf{a} \cdot \mathbf{b}$. Transverse coordinates $\mathbf{x}$ and $\mathbf{y}$ will hereafter be restricted to values corresponding to transverse lattice sites. The covariant derivative (4) in direction $x^{\alpha}$ becomes

$$
\bar{D}_{\alpha} M_{r}(\mathbf{x})=\left[\partial_{\alpha}+i A_{\alpha}(x)\right] M_{r}(\mathbf{x})-i M_{r}(\mathbf{x}) A_{\alpha}(x+a \hat{r})
$$

in the partial continuum limit. One finds a transverse lattice action

$$
\begin{aligned}
S= & \int d x^{0} d x^{3} \sum_{\mathbf{x}}\left(\operatorname{Tr}\left\{\bar{D}_{\alpha} M_{r}(\mathbf{x})\left(\bar{D}^{\alpha} M_{r}(\mathbf{x})\right)^{\dagger}\right\}\right. \\
& \left.-\frac{1}{2 G^{2}} \operatorname{Tr}\left\{F_{\alpha \beta} F^{\alpha \beta}\right\}-V_{\mathbf{x}}[M]\right),
\end{aligned}
$$

where we have chosen to normalize $M_{r}(\mathbf{x})$ to have a canonical kinetic term and repeated indices are summed unless stated otherwise. The dimensionful coupling $G^{2}(a)$ is such that $a^{D-2} G^{2} \rightarrow g_{D}^{2}$ in the classical continuum limit $a \rightarrow 0$, where $g_{D}^{2}$ is the continuum gauge coupling in $D$ dimensions. If we keep only Wilson loops and their products of total length 2 and 4 (which we justify in the next section), the effective potential $V_{\mathbf{x}}$ generically consists of Wilson loops among those shown in Fig. 1, but now understood to be in the remaining $(D-2)$-dimensional transverse lattice and pinned at one point to $\mathbf{x}$.

The basis (6) for an effective QCD light-front Hamiltonian was earlier proposed in Ref. [2], motivated by considerations of two-dimensional sigma models. Subsequently, an exploratory glueball calculation was performed [3] for some simple choices of $V$. Those authors speculated that one might be able to find a scaling trajectory in $V$, corresponding to QCD. We have extended the methods of Ref. [3] in order to answer this question and perform in this paper quantitative first-principles calculations. This allows us to confirm the existence of an approximate scaling trajectory at large transverse lattice spacing (at least in $2+1$ dimensions) and show that it yields the same results for physical observables that are available from conventional methods of studying QCD.

As mentioned above, to take best advantage of the simplifications in the light front afforded by linear link variables $M_{r}(\mathbf{x})$, it is quite important to be able to quantize about $M_{r}(\mathbf{x})=0$. Our calculations therefore must implicitly test a light-front Hamiltonian version of the color-dielectric regime: In the light-front Hamiltonian limit of lattice action $S$, we search for a scaling trajectory by canonical quantization about $M_{r}(\boldsymbol{x})=0$, taken as the true ground state.

Once outside of the purported color-dielectric regime, when $a$ is sufficiently small and $M_{r}(\mathbf{x})=0$ is no longer the minimum of energy, we expect the zero-mode structure of light-front quantization to become much more complicated [17].

\section{CANONICAL LIGHT-FRONT QUANTIZATION}

\section{A. Cutoffs and gauge invariance}

We introduce light-front coordinates $x^{ \pm}=x_{\mp}=\left(x^{0}\right.$ $\left.\pm x^{D-1}\right) / \sqrt{2}$, for all two-vectors in the $\left(x^{0}, x^{D-1}\right)$ plane, and quantize by treating $x^{+}$as canonical time. The variables conjugate to $x^{ \pm}$and $\mathbf{x}$ are $k^{\mp}=\left(k^{0} \mp k^{D-1}\right) / \sqrt{2}$ and $\mathbf{k}$, respectively. Particles and antiparticles have $k^{+} \geqslant 0$. The transverse lattice theory will have divergences associated with the $k^{+}$ $\rightarrow 0$ limit. This is a high $k^{-}$light-front energy limit, as follows from the dispersion relation

$$
k^{-}=\frac{\mu^{2}+|\mathbf{k}|^{2}}{2 k^{+}}
$$

for free particles of mass $\mu$. At finite transverse lattice spacing $a$, this is the only source of divergence, since $\mathbf{k}$ is bounded by the Brillouin zone $\pi / a$. In fact, the theory is just a set of coupled $(1+1)$-dimensional continuum field theories, and there are only the mild free-field divergences characteristic of two-dimensional superrenormalizable theories. For pure gauge theory, finiteness is ensured by a suitable normal-ordering prescription.

At the level of free fields, it follows that if $\mu^{2}>0$, the quantum vacuum is free of particles. Because the light-front momentum $k^{+} \geqslant 0$ and is conserved, the zero-momentum vacuum state with even one massive $\left(k^{+}=0\right)$ particle present is infinitely higher in energy than one with no particles. In the interacting theory, the particles must not condense either. In other words, one assumes that one is already expanding about the true vacuum state and all condensation effects are parametrized by effective interactions in the Hamiltonian. This scenario is precisely realized in the colordielectric regime; the dielectric fields $M_{r}(\mathbf{x})$ are massive and $M_{r}(\mathbf{x})=0$ is the true vacuum. The nontrivial problem is to demonstrate a coupling trajectory in this regime which restores all fundamental (unbroken) symmetries of the theory that have been broken by the regulators.

For fixed total momentum $P^{+}$, a large number of particles must carry a very large light-front energy, since each particle is forced to carry a small positive $k^{+}$. Although this would also be true of massive particles in an equal-time quantization, the effect is much more pronounced in the light-front formulation, because of the peculiar inverse relation between momentum and energy (7). In particular, one notes that in the effective potential $V$, higher powers of the field $M_{r}(\mathbf{x})$ would couple precisely to those components of the wave function with a large number of $M$-quanta. This is our physical justification for truncation of $V$ to the shortest Wilson loops. Higher loops may be added as greater accuracy is required in the low-energy theory.

To regulate the $k^{+}=0$ region, we will impose periodic boundary conditions in $x^{-}$[18], with period $\mathcal{L}$, taking the longitudinal momentum space continuum limit $\mathcal{L} \rightarrow \infty$ for fixed $a$. This gauge-invariant discretization is also a useful starting point for the numerical investigation of the lightfront Hamiltonian [19] [discrete light-cone quantization (DLCQ)].

The theory (6) possesses the fields $M_{r}\left(x^{+}, x^{-}, \mathbf{x}\right)$, 
$A_{+}\left(x^{+}, x^{-}, \mathbf{x}\right)$, and $A_{-}\left(x^{+}, x^{-}, \mathbf{x}\right)$, together with the unitary gauge symmetry at each transverse lattice site $\mathbf{x}$ :

$$
\begin{aligned}
& A_{\alpha}(\mathbf{x}) \rightarrow U^{\dagger}(\mathbf{x}) A_{\alpha}(\mathbf{x}) U(\mathbf{x})+i\left(\partial_{\alpha} U^{\dagger}(\mathbf{x})\right) U(\mathbf{x}) \\
& M_{r}(x) \rightarrow U^{\dagger}(\mathbf{x}) M_{r}(\mathbf{x}) U(\mathbf{x}+a \hat{r}) .
\end{aligned}
$$

We choose the light-front gauge ${ }^{2} A_{-}=0$ and eliminate the resulting constrained field $A_{+}(\mathbf{x})$ by solving its equation of motion classically [3] at each site $\mathbf{x}$ :

$$
\frac{2}{G^{2}} \partial_{-}^{2} A_{+}=J^{+},
$$

where

$$
\begin{aligned}
J^{+}(\mathbf{x})= & i\left[M_{r}(\mathbf{x}) \partial_{-} M_{r}^{\dagger}(\mathbf{x})-\partial_{-} M_{r}(\mathbf{x}) M_{r}^{\dagger}(\mathbf{x})\right. \\
& +M_{r}^{\dagger}(\mathbf{x}-a \hat{r}) \partial_{-} M_{r}(\mathbf{x}-a \hat{r})-\partial_{-} M_{r}^{\dagger}(\mathbf{x}-a \hat{r}) \\
& \left.\times M_{r}(\mathbf{x}-a \hat{r})\right] .
\end{aligned}
$$

The charges $\widetilde{J}^{+}(0, \mathbf{x})=\int d x^{-} J^{+}\left(x^{-}, \mathbf{x}\right)$ generate $x^{-}$-independent gauge transformations before gauge fixing, and the requirement that they vanish for finite-energy states is the statement of color confinement for the transverse lattice formulation at fixed $a$ [3]. The mechanism for the confining linear potential in longitudinal and transverse directions is somewhat different in each case, however. For the longitudinal direction it arises as the Coulomb potential for the $(1+1)$-dimensional gauge field theory at each site $\mathbf{x}$. In the transverse directions it arises through the Wilson-KogutSusskind argument for lattice gauge theories [11,21]. For a color singlet at each $\mathbf{x}$, we must build a color string with the (massive) $M_{r}(\mathbf{x})$ link fields, the energy being proportional to the number of links in the string. Note, however, that the color-singlet requirement came from the continuum longitudinal dynamics. In later sections, we will show how one can verify these intuitive arguments by explicit calculation of the transverse and longitudinal string tensions.

\section{B. Energy and momentum}

Only $M_{r}(\mathbf{x})$ now remains as a physical degree of freedom. We derive the total light-front momentum and energy $P^{\mu}=\int d x^{-} \Sigma_{\mathbf{x}} \theta^{+\mu}$,

$$
P^{+}=2 \int d x^{-} \sum_{\mathbf{x}, r} \operatorname{Tr}\left\{\partial_{-} M_{r}(\mathbf{x}) \partial_{-} M_{r}(\mathbf{x})^{\dagger}\right\},
$$

\footnotetext{
${ }^{2}$ We have shown that the associated zero mode $\int d x^{-} A_{-}$may be neglected in our calculations.
}

$$
\begin{aligned}
P^{-}= & \int d x^{-} \sum_{\mathbf{x}} V_{\mathbf{x}}[M] \\
& -\frac{G^{2}}{4}\left(\operatorname{Tr}\left\{J^{+}(\mathbf{x}) \frac{1}{\partial_{-}^{2}} J^{+}(\mathbf{x})\right\}\right. \\
& \left.-\frac{1}{N} \operatorname{Tr}\left\{J^{+}(\mathbf{x})\right\} \frac{1}{\partial_{-}^{2}} \operatorname{Tr}\left\{J^{+}(\mathbf{x})\right\}\right) .
\end{aligned}
$$

The $1 / N$ correction to the Coulomb term in Eq. (13) is necessary at large $N$ for $\mathrm{SU}(N)$ to ensure that the longitudinal gluon $A_{+}$, having no color-singlet part, does not couple to gauge singlet states. It will turn out to be important only for two-link states at nonzero transverse momentum $\mathbf{P}$.

The residual local gauge symmetry, generated by $\widetilde{J}^{+}(0, \mathbf{x})$, is

$$
M_{r}(\mathbf{x}) \rightarrow U^{\dagger}(\mathbf{x}) M_{r}(\mathbf{x}) U(\mathbf{x}+a \hat{r}),
$$

with $U(\mathbf{x})$ independent of $x^{-}$. The light-front energy (13) is not finite unless the associated charge vanishes at each site $\mathbf{x}: \widetilde{J}^{+}(0, x)=0$. Thus only states invariant under the residual gauge symmetry (14) will have finite energy.

\section{Fock space}

We impose canonical commutation relations at $x^{+}=0$ :

$$
\begin{aligned}
& {\left[M_{r, i j}\left(x^{-}, \mathbf{x}\right),\left(\partial_{-} M_{s, k l}\left(y^{-}, \mathbf{y}\right)\right)^{\dagger}\right]} \\
& \quad=\frac{1}{2} \delta_{i l} \delta_{j k} \delta\left(x^{-}-y^{-}\right) \delta_{\mathbf{x}, \mathbf{y}} \delta_{r, s} .
\end{aligned}
$$

We work in longitudinal momentum space and transverse position space:

$$
\begin{aligned}
M_{r}\left(x^{+}=0, x^{-}, \mathbf{x}\right)= & \frac{1}{\sqrt{4 \pi}} \int_{0}^{\infty} \frac{d k}{\sqrt{k}}\left[a_{-r}(k, \mathbf{x}) e^{-i k x^{-}}\right. \\
& \left.+a_{r}^{\dagger}(k, \mathbf{x}+a \hat{r}) e^{i k x^{-}}\right] \\
{\left[a_{\lambda, i j}(k, \mathbf{x}),\left(a_{\rho, k l}(\widetilde{k}, \mathbf{y})\right)^{\dagger}\right]=} & \delta_{i k} \delta_{j l} \delta_{\lambda \rho} \delta_{\mathbf{x}, \mathbf{y}} \delta(k-\widetilde{k}), \\
{\left[a_{\lambda, i j}(k, \mathbf{x}), a_{\rho, k l}(\widetilde{k}, \mathbf{y})\right]=} & 0 .
\end{aligned}
$$

Here $\left(a_{\lambda, i j}\right)^{\dagger}=\left(a_{\lambda}^{\dagger}\right)_{j i}$ and $\lambda, \rho \in\{ \pm 1, \ldots \pm(D-2)\}$. For clarity, we omit the + superscript on light-front momentum $k^{+}$ hereafter. The Fock vacuum $|0\rangle$ satisfies $: P^{-}:|0\rangle=: P^{+}:|0\rangle$ $=0$ and is the physical vacuum. The Fock space operator $a_{\lambda}^{\dagger}(k, \mathbf{x})$ creates a mode with longitudinal momentum $k$ on the link emanating from site $\mathbf{x}$ in transverse direction $\hat{\lambda}$. This Fock space is already diagonal in $P^{+}$and serves as a basis for finding the energy levels of $P^{-}$. Since the glueball boundstates do not interact with one another at $N=\infty$, this in turn will diagonalize the invariant mass operator $\mathcal{M}^{2}$ $=2 P^{+} P^{-}-\mathbf{P}^{2}$ in the frame $\mathbf{P}=0$.

The combinations singlet under Eq. (14) are the "string", states which form closed flux loops on the transverse lattice, for example, 


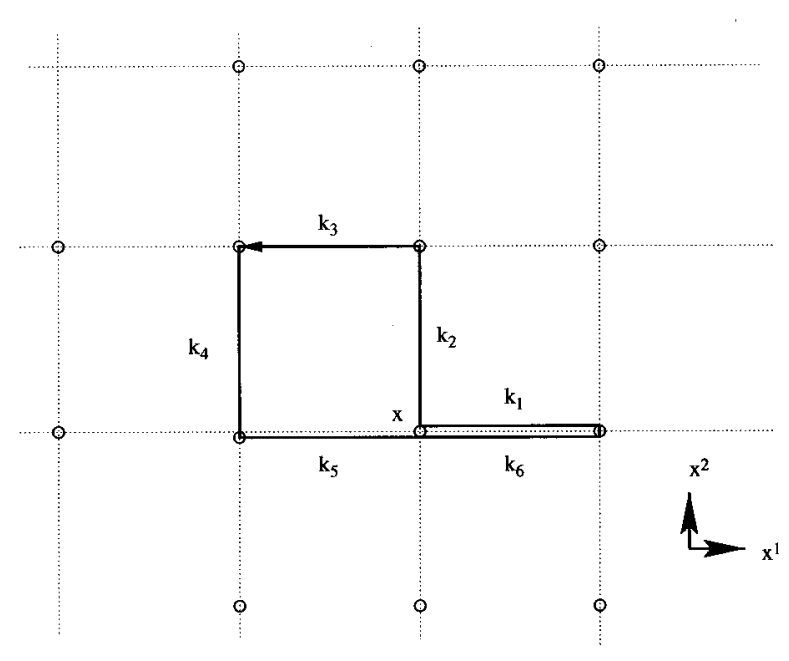

FIG. 2. An example of a length of six loops on the transverse lattice, showing also the longitudinal momentum carried by each link, corresponding to the Fock state (19).

$$
\begin{aligned}
\operatorname{Tr}\{ & a_{-1}^{\dagger}\left(k_{1}, \mathbf{x}+a \hat{1}\right) a_{2}^{\dagger}\left(k_{2}, \mathbf{x}\right) a_{-1}^{\dagger}\left(k_{3}, \mathbf{x}+a \hat{2}\right) \\
& \left.\quad \times a_{-2}^{\dagger}\left(k_{4}, \mathbf{x}+a \hat{2}-a \hat{1}\right) a_{1}^{\dagger}\left(k_{5}, \mathbf{x}-a \hat{1}\right) a_{1}^{\dagger}\left(k_{6}, \mathbf{x}\right)\right\}|0\rangle,
\end{aligned}
$$

as illustrated in Fig. 2. The longitudinal momenta $k_{m}$ are unconstrained except for $k_{m}>0$ and conservation, $\sum_{m=1}^{6} k_{m}$ $=P^{+}$in the above example.

\section{STRUCTURE FUNCTIONS}

A standard gauge-invariant definition in the continuum of the gluon distribution function $G(x)$ in a hadron $\left|\Psi\left(P^{+}\right)\right\rangle$is [22]

$$
\begin{aligned}
G(x)= & \frac{1}{2 \pi x P^{+}} \int d y^{-} e^{-i x P^{+} y^{-}}\left\langle\Psi\left(P^{+}\right)\right| F_{a}^{+r}\left(0, y^{-}, \mathbf{0}\right) \\
& \times \mathcal{O}_{a b} F_{b}^{+r}(0,0, \mathbf{0})\left|\Psi\left(P^{+}\right)\right\rangle,
\end{aligned}
$$

where $F_{a}^{\mu \nu}\left(x^{+}, x^{-}, \mathbf{x}\right)$ is the field strength tensor with color index $a, b, c \in\left\{1, \ldots, N^{2}-1\right\}$ and

$$
\mathcal{O}=\mathcal{P} \exp \left(i \int_{0}^{y^{-}} d z^{-} A_{c}^{+}\left(0, z^{-}, \mathbf{0}\right) t_{c}\right) .
$$

Here the $t_{c}$ are the $\mathrm{SU}(N)$ generators in the adjoint representation. In the light-front gauge $A_{-}=0$ this definition (20) reduces to the expectation value of the gluon number operator. Note that the answer is in fact independent of the choice of $\mathbf{x}$. Since the color-dielectric formulation deals with a set of collective variables rather than with gluons, the transformation from one to the other being unknown explicitly, we cannot directly measure the gluon distribution. We recall however from Eqs. (4) and (5) that $\bar{D}_{-} M_{r}(\mathbf{x})$ reduces to the field strength $F_{-r}$ in the continuum limit. It seems sensible therefore to make the substitution $F_{-r} \rightarrow \bar{D}_{-} M_{r}(\mathbf{x})$ in the definition (20). We call the resulting gauge-invariant distri- bution $G_{d}$ to avoid confusion. In the continuum limit $G_{d}$ reduces to $G(x)$, but in general they are different. Since $M_{r}(\mathbf{x})$ are lattice variables, $G_{d}$ will evolve with the renormalization scale defined by the edge of the Brillouin zone $\Lambda_{\perp}=\pi / a$. In the light-front gauge $A_{-}=0$, we have

$$
\begin{aligned}
G_{d}\left(x, \Lambda_{\perp}\right)= & \frac{1}{2 \pi x P^{+}} \int d y^{-} e^{-i x P^{+} y^{-}}\left\langle\Psi\left(P^{+}\right)\right| \\
& \times \operatorname{Tr}\left\{\partial_{-} M_{r} \partial_{-} M_{r}^{\dagger}\right\}\left|\Psi\left(P^{+}\right)\right\rangle \\
= & \left\langle\Psi\left(P^{+}\right)\left|a_{\lambda, i j}^{\dagger}\left(x P^{+}, \mathbf{x}\right) a_{\lambda, i j}\left(x P^{+}, \mathbf{x}\right)\right| \Psi\left(P^{+}\right)\right\rangle .
\end{aligned}
$$

Thus $G_{d}$ has a simple interpretation: the expectation value of the number operator for link fields of longitudinal momentum fraction $x$. It trivially satisfies the momentum sum rule

$$
\int_{0}^{1} d x x G_{d}(x)=1
$$

and the modes of $M_{r}(\mathbf{x})$ may be interpreted as "fat" partons with finite transverse extent. Bjorken scaling violations due to this finite extent will manifest themselves through the evolution with $\Lambda_{\perp}$ along the scaling trajectory. From the discussion of Sec. II A, one expects the scaling trajectory to pass from large to small values of parton mass $\mu^{2}$ as the lattice spacing $a$ is reduced. Since $a$ is a measure of the transverse size of the parton, the parton becomes fatter as it becomes heavier. As $\Lambda_{\perp}$ is increased ( $a$ decreased), the glue distribution $G_{d}$ is expected to evolve to smaller $x$. This follows simply from the fact that the small- $x$ region becomes enhanced the lighter the parton masses, since the free lightfront kinetic energy (7) is $\sim \mu^{2} / x$ for a parton of momentum fraction $x$. We explicitly calculate structure functions later.

\section{LARGE- $N$ LIMIT}

In pure gauge theory, the corrections to the large- $N$ limit usually form an asymptotic series in $1 / N^{2}$. For our problem, a dramatic simplification of the relevant Fock space takes place when one neglects these corrections. First, in Ref. [9] and I, it was shown that in the color-dielectric regime, the Hilbert space of $\mathbf{P}=0$ Wilson loops on the transverse lattice and the light-front Hamiltonian which acts upon them undergo dimensional reduction; they may be replaced by an equivalent problem where all links in a given transverse direction are identified. One effectively works on a transverse lattice with one link in each direction and periodic boundary conditions. This is a manifestation of Eguchi-Kawai reduction [23] in the light-front Hamiltonian framework. One may drop the argument $\mathbf{x}$ in all previous expressions:

$$
M_{r}\left(x^{-}, \mathbf{x}\right) \rightarrow M_{r}\left(x^{-}\right), \quad a_{\lambda}(k, \mathbf{x}) \rightarrow a_{\lambda}(k) .
$$

The proof of the dimensional reduction (25) does not encounter any phenomena analogous to center U(1) breaking [24]; however, one must recall that the color-dielectric regime, on which the proof is based, is only expected at large 
transverse lattice spacing. In this way, in $D$ spacetime dimensions one ends up having to solve two-dimensional large- $N$ gauge theories coupled to $2^{D-2}$ complex adjoint scalars. The light-front quantization of this kind of two-dimensional gauge theory has recently been developed in Ref. [20], whose mathematical analysis we follow closely. ${ }^{3}$

The Hilbert space is further simplified by the fact that the light-front Hamiltonian propagates Wilson loops without splitting or joining at large $N$ [25]; the amplitude for this is order $1 / N$. Thus the light-front Hamiltonian may be studied in the space of connected Wilson loops, that is, states containing one color trace, for example, Eq. (19).

The explicit light-front Hamiltonian (13) for the frame $\mathbf{P}$ $=0$ now becomes, equivalently $(D=4)$,

$$
\begin{aligned}
P^{-}= & \int d x^{-}-\frac{G^{2}}{4} \operatorname{Tr}\left\{J^{+} \frac{1}{\partial_{-}^{2}} J^{+}\right\}-\frac{\beta}{N a^{D-2}} \operatorname{Tr}\left\{M_{2}^{\dagger} M_{1}^{\dagger} M_{2} M_{1}+\text { H.c. }\right\}+\sum_{r} \mu^{2} \operatorname{Tr}\left\{M_{r} M_{r}^{\dagger}\right\}+\sum_{r} \frac{\lambda_{1}}{a^{D-2} N} \operatorname{Tr}\left\{M_{r} M_{r}^{\dagger} M_{r} M_{r}^{\dagger}\right\} \\
& +\frac{\lambda_{2}}{a^{D-2} N} \sum_{r} \operatorname{Tr}\left\{M_{r} M_{r} M_{r}^{\dagger} M_{r}^{\dagger}\right\}+\sum_{r} \frac{\lambda_{3}}{a^{D-2} N^{2}}\left(\operatorname{Tr}\left\{M_{r} M_{r}^{\dagger}\right\}\right)^{2} \\
& +\frac{\lambda_{4}}{a^{D-2} N} \sum_{\sigma= \pm 2, \sigma^{\prime}= \pm 1} \operatorname{Tr}\left\{M_{\sigma}^{\dagger} M_{\sigma} M_{\sigma^{\prime}}^{\dagger} M_{\sigma^{\prime}}\right\}+\frac{4 \lambda_{5}}{a^{D-2} N^{2}} \operatorname{Tr}\left\{M_{1} M_{1}^{\dagger}\right\} \operatorname{Tr}\left\{M_{2} M_{2}^{\dagger}\right\} \\
J^{+}= & i\left(M_{r} \overleftrightarrow{\partial}_{-} M_{r}^{\dagger}+M_{r}^{\dagger} \overleftrightarrow{\partial}_{-} M_{r}\right)
\end{aligned}
$$

We will later show how $\mathbf{P} \neq 0$ may be treated in the same dimensionally reduced framework, by adding appropriate phase factors to matrix elements of Eq. (26) in the dimensionally reduced Fock basis. We have displayed in Eq. (26) terms of the effective potential corresponding to Fig. 1 on the transverse lattice, with coefficients $\mu^{2}, \lambda_{1}, \lambda_{2}, \lambda_{3}, \lambda_{4}, \lambda_{5}$, and $\beta$. These parameters are to be fixed by a scaling analysis. Note that the multiple Trace operators coupling to $\lambda_{3}$ and $\lambda_{5}$ do contribute in leading order of $N$, but only on two-parton Fock states. The dimensional reduction in the large- $N$ limit was not discussed by the authors of Refs. [2, 3], but was used implicitly in the calculations performed there.

The fact that the large- $N$ limit of the light-front Hamiltonian simplifies the calculations of physical observables in this way is to be contrasted with other quantization schemes. A path integral evaluation of Green's functions

$$
\left\langle G\left[A_{\mu}\right]\right\rangle=\int D A_{\mu} G\left[A_{\mu}\right] \exp \left(-\int d x S\left[A_{\mu}\right]\right)
$$

should be dominated exactly at $N=\infty$ by an $x$-independent classical saddle point configuration of the fields $A_{\mu}$, the "master field" [26]. This is motivated by the factorization property

$$
\begin{aligned}
\left\langle\operatorname{Tr}\left\{A_{\mu_{1}} A_{\mu_{2}} \cdots A_{\mu_{n}}\right\} \operatorname{Tr}\left\{A_{\mu_{n+1}} A_{\mu_{n+2}} \cdots A_{\mu_{m}}\right\}\right\rangle & =\left\langle\operatorname{Tr}\left\{A_{\mu_{1}} A_{\mu_{2}} \cdots A_{\mu_{n}}\right\}\right\rangle\left\langle\operatorname{Tr}\left\{A_{\mu_{n+1}} A_{\mu_{n+2}} \cdots A_{\mu_{m}}\right\}\right\rangle \\
& +\frac{1}{N^{2}}\left\langle\operatorname{Tr}\left\{A_{\mu_{1}} A_{\mu_{2}} \cdots A_{\mu_{n}}\right\} \operatorname{Tr}\left\{A_{\mu_{n+1}} A_{\mu_{n+2}} \cdots A_{\mu_{m}}\right\}\right\rangle_{c},
\end{aligned}
$$

\footnotetext{
${ }^{3}$ The dimensional reduction discussed in Ref. [20], which is an approximation, is not to be confused with the large- $N$ reduction discussed here, which is exact.
}

where $\langle\cdots\rangle_{c}$ is the connected amplitude. Equation (29) can be understood by the usual planar diagram counting rules [6], which attach a factor $N^{2-2 G-P}$ to a diagram with $G$ handles and $P$ boundaries. The expectation value of two Wilson loops has a term of order $N^{2}$, from the disconnected disks ( $P=1$ for each) covering each loop, plus a term of order $N^{0}$ from the connected amplitude, the cylinder connecting the two loops. Thus at $N=\infty$, where Eguchi-Kawai reduction in the lattice theory takes place, it appears that one can only calculate disconnected Green's functions and the vacuum energy. In an equal-time Hamiltonian approach, eigenvalues are also dominated by the vacuum contribution of order $N^{2}$ (amplitude $N$ to create or annihilate a zeromomentum Wilson loop from the vacuum). It is therefore not possible to extract the physical spectrum at $N=\infty$, since this involves contributions down by order $1 / N^{2}$ compared to the vacuum contribution. Physical states interact with the vacuum by emitting or absorbing zero-momentum Wilson loops with amplitude of order $N^{0}$ (amplitude $1 / N$ to split or join and amplitude $N$ to emit or absorb from the vacuum).

It is then clear why the transverse lattice light-front Hamiltonian framework is uniquely suited for calculations of observables-as opposed to vacuum structure-in the large- $N$ limit. The triviality of the light-front vacuum implies that there is no order $N^{2}$ contribution. Nontrivial contributions begin at order $N^{0}$, corresponding to the free-string-like propagation of a Wilson loop. In this way the spectrum of physical states is directly computable at $N=\infty$, where a dimensional reduction in the number of degrees of freedom takes place in the lattice theory. However, unlike the path integral vacuum problem where a reduction to zero dimensions takes place, in the light-front transverse lattice approach dimensional reduction takes place only in the transverse directions. The statement of a large- $N$ (Eguchi-Kawai) reduction in $D$ spacetime dimensions for physical observ- 
ables, rather than vacuum structure, is that one can solve for observables in a field theory of $D-2$ fewer dimensions in the large-N limit.

\section{STRING TENSIONS}

To measure the string tension $\sigma_{T}$ in the $\mathbf{x}=\left(x^{1}, x^{2}\right)$ directions, consider a transverse lattice with $\mathbf{n}=\left(L_{1} / a, L_{2} / a\right)$ links and periodic boundary conditions. We construct a basis of Polyakov-loop winding modes in Fock space in the frame $\mathbf{P}=0$ that wind once around this lattice in both the $x^{1}$ and $x^{2}$ directions. The shortest such loop has length $|\mathbf{n}|$ in lattice units. One may extract from the lowest invariant-mass eigenvalue $\mathcal{M}^{2}$ the lattice string tension $a \sigma_{T}$ via

$$
\mathcal{M}^{2} \approx a^{2} \sigma_{T}^{2}|\mathbf{n}|^{2}
$$

for sufficiently large $|\mathbf{n}|$. The form (30) will be imposed as one of the scaling requirements; that is, $\sigma_{T}$ must not vary with $a$ or direction $\mathbf{n} /|\mathbf{n}|$. Because of the large- $N$ reduction, this procedure is equivalent to using Polyakov loops $W_{\mathbf{n}}$ of winding number $\mathbf{n}$, on the transverse lattice with one link in each direction. The total winding number in each direction is conserved due to center symmetry. In the large- $N$ limit, we study single Polyakov loops for the same reason that splitting of Wilson loops $W_{(0,0)}$ is suppressed by $1 / N$. Thus $\mathbf{n}$ labels disconnected sectors of the Hilbert space.

In sectors of nonzero winding $|\mathbf{n}| \neq 0$, there are further operators one could add to the effective potential $V[M]$ at the same level of approximation. They will in general be necessary for finiteness, as well as to achieve scaling in these sectors. In particular, when the Hamiltonian (26) is put into normal order, two kinds of quadratic terms are generated, with divergent coefficients $c_{m}$ and $c_{w}$, in the $\mathcal{L} \rightarrow \infty$ limit:

$$
\begin{gathered}
c_{m} \int_{0}^{\infty} \frac{d k}{k} \operatorname{Tr}\left\{a_{\lambda}^{\dagger}(k) a_{\lambda}(k)\right\}, \\
\frac{c_{w}}{N} \int_{0}^{\infty} \frac{d k}{k} \operatorname{Tr}\left\{a_{\lambda}^{\dagger}(k)\right\} \operatorname{Tr}\left\{a_{\lambda}(k)\right\} .
\end{gathered}
$$

The first (31) is a link-field mass self-energy and is dealt with by straightforward mass renormalization, leaving the finite part $\mu^{2}$. The second (32) is suppressed by $1 / N$ on all states except $\operatorname{Tr}\left\{a_{\lambda}^{\dagger}\left(P^{+}\right)\right\}$, the one-parton state of winding number $\hat{\lambda}$. To avoid the divergence of the mass of this state, one must add a new counterterm to $V[M]$ given by

$$
-\frac{1}{N} \operatorname{Tr}\left\{M_{r}\right\} \operatorname{Tr}\left\{M_{r}^{\dagger}\right\}
$$

with an appropriate coefficient to cancel $c_{w}$. It is then possible to have a finite part left over, which will appear as a new parameter in the theory. In this case one loses one unit of predictive power from the measurement of energy levels in the winding number \pm 1 sector. Evidently, there are many similar multiple trace operators one can add to the Hamiltonian, which act in this way on $\mathbf{n} \neq 0$ sectors. They are quite analogous to the multiple trace operators, of which $\lambda_{3}$ is an example (26), which can appear in the $\mathbf{n} \neq 0$ sector (in that case, winding number is conserved "in each trace"). However, (33) is the only one required on the grounds of finiteness when the Hamiltonian (26) is put into normal order. In a winding $\mathbf{n}$ sector, multiple trace operators that contribute in leading order of $N$ must contain at least $\Sigma_{r}\left|n^{r}\right|$ powers of $M_{r}(\mathbf{x})$. Since we try to extract $\sigma_{T}$ from large $|\mathbf{n}|$, we will neglect this possible proliferation of parameters to a first approximation, since we neglect higher powers of $M_{r}(\mathbf{x})$ in the effective potential $V[M]$.

In addition to using Polyakov loops of nonzero winding number in the transverse direction, we can measure the string tension using the heavy-quark potential [10]. The advantage of this approach is that the heavy-quark potential can be measured in the $x^{D-1}$ as well as in the transverse directions. The main disadvantage is that this quantity is numerically difficult to compute. Our procedure for measuring this potential is described in Appendix A. For sources separated by distance $L$ in direction $x^{3}$ only, we extract the longitudinal string tension $\sigma_{L}$ by fitting the energy to $\sigma_{L} L$ for large $L$. Of course, $\sigma_{L}=\sigma_{T}$ in a rotationally invariant theory, and in fact we will impose this condition as one of the scaling requirements.

\section{LORENTZ COVARIANCE}

One of the advantages of light-front quantization is that boosts-both longitudinal and transverse-become simple. However, our transverse lattice regulator, along with our use of effective degrees of freedom, breaks transverse Lorentz covariance. Instead, we will demand Lorentz covariance under transverse boosts for the lightest glueball states; we will use this to help determine the effective potential.

Let us introduce states of nonzero transverse momentum $\mathbf{P} \neq 0$. A generic $p$-link state of transverse momentum $P$ has the form

$$
\begin{aligned}
\left|\Psi\left(P^{+}, \mathbf{P}\right)\right\rangle= & \frac{1}{\sqrt{N^{p}}} \sum_{\mathbf{y}} e^{i \mathbf{P} \cdot(\mathbf{y}+\overline{\mathbf{x}})} \operatorname{Tr}\left\{a_{\lambda_{1}}^{\dagger}\left(k_{1}, \mathbf{x}_{1}+\mathbf{y}\right)\right. \\
& \left.\times a_{\lambda_{2}}^{\dagger}\left(k_{2}, \mathbf{x}_{2}+\mathbf{y}\right) \cdots a_{\lambda_{p}}^{\dagger}\left(k_{p}, \mathbf{x}_{p}+\mathbf{y}\right)\right\}|0\rangle,
\end{aligned}
$$

where the winding number vanishes $\sum_{i=1}^{p} \hat{\lambda}_{i}=0$ and $\mathbf{y}$ is summed over links of the transverse lattice. The transverse coordinates must obey the formula

$$
\mathbf{x}_{i}=\mathbf{x}_{i-1}+a \hat{\lambda}_{i-1}, \quad i<1 \leqslant p .
$$

The overall phase $\overline{\mathbf{x}}$ is a convention. Although we have, for the sake of clarity, shown the transverse coordinates $\mathbf{x}$ for the link fields $a_{\lambda}^{\dagger}(k, \mathbf{x})$, in Appendix B we show that they may be dropped; a nonzero transverse momentum is equivalent to introducing various phase factors in the Hamiltonian in the Eguchi-Kawai reduced theory.

We now indicate how a scaling analysis can be carried out with these states, by enforcing $\mathrm{SO}(3,1)$ Lorentz invariance of physical observables. There are a number of relativistic cri- 
teria which one might apply. With only the spectrum of glueball mass ratios, as a function of the couplings in $V[M]$, one might try to obtain Lorentz multiplets. This is a rather narrow test, however, since it pays no attention to scalar bound states. A better measure of relativistic covariance is the glueball dispersion formula. Perfectly relativistic glueballs should satisfy

$$
2 P^{+} P^{-}=\mathcal{M}^{2}+|\mathbf{P}|^{2} .
$$

Transverse lattice glueballs will in general satisfy

$$
2 P^{+} P^{-}=G^{2} N\left(\mathcal{M}_{0}^{2}+\mathcal{M}_{1}^{2} a^{2}|\mathbf{P}|^{2}+\mathcal{M}_{2}^{2} a^{4}|\mathbf{P}|^{4}+\cdots\right) .
$$

We have used $G^{2} N$ as the overall scale, so that the measurable coefficients $\mathcal{M}_{i}^{2}$ above depend only upon the dimensionless ratios ( $\tau$ is introduced in Appendix A)

$$
\begin{gathered}
m^{2}=\frac{\mu^{2}}{G^{2} N}, \quad l_{i}=\frac{\lambda_{i}}{a^{D-2} G^{2} N}, \quad t=\frac{\tau}{\sqrt{G^{2} N} a^{D-3}}, \\
b=\frac{\beta}{a^{D-2} G^{2} N} .
\end{gathered}
$$

In order that Eq. (37) agree with Eq. (36), one can tune the couplings so that $\mathcal{M}_{2}^{2}=0$ and $a^{2} G^{2} N \mathcal{M}_{1}^{2}=1$ for each glueball in the low-lying spectrum. Because of the fact that there are transverse lattice hopping terms in $P^{-}$, even when $V$ $=0$, the dispersion relation automatically becomes asymptotically quadratic for small $a \mathbf{P}$. For this reason, the condition $\mathcal{M}_{2}^{2}=0$ is more difficult to use to fix $V$. The condition $a^{2} G^{2} N \mathcal{M}_{1}^{2}=1$, which is more useful, ensures that the speed of light is isotropic. The rotational invariance of the speed of light in the transverse directions was discussed in Ref. [3]. In order to ensure that the speed of light in transverse directions $c_{T}$ equals that in the $x^{D-1}$ direction $c_{L}$ (set to 1 by convention), one must determine the dimensionless combination $a^{2} G^{2} N$

One way is to measure both the longitudinal and transverse string tensions. This also allows one to express the glueball spectrum in terms of the string tension which is essential for making an absolute prediction of the glueball masses. Suppose we make the following three measurements of dimensionful quantities:

$$
\begin{aligned}
& v_{1} G^{2} N=c \sigma, \\
& v_{2} G^{2} N=a^{2} \sigma^{2}, \\
& v_{3} G^{2} N=\sigma,
\end{aligned}
$$

where $v_{1}, v_{2}, v_{3}$ are measured dimensionless numbers, $c$ is an unknown constant, and $\sigma$ is the continuum string tension. Equation (39) comes from a glueball mass measurement. Equation (40) comes from the transverse winding spectrum, while Eq. (41) comes from the asymptotic heavy-source potential in the $x^{D-1}$ direction. We have also demanded that the longitudinal and transverse tensions be equal, $\sigma_{T}=\sigma_{L}$ $=\sigma$. Scaling means that the right-hand sides of the above equations are not multiplied by dimensionless functions of $a^{2} G^{2} N$. We now show that this is enough to solve the theory completely.

The slope of the dispersion relations at $\mathbf{P}=0$ for a few low-lying glueballs is used to estimate the scaling trajectory of $V$, by demanding isotropy of the speed of light. From Eqs. (36) and (37) one needs to determine $\mathrm{G}^{2} \mathrm{Na}^{2}$ in order to compare transverse and longitudinal scales when comparing the speeds of light. From Eqs. (40) and (41) we can deduce that

$$
G^{2} N a^{2}=v_{2} / v_{3}^{2}
$$

There are a number of self-consistency checks one can perform, such as examining deviations from quadratic dispersion (nonzero $\mathcal{M}_{2}^{2}$ ), the Lorentz-multiplet structure in the glueball spectrum, and rotational invariance of the heavysource potential. Similarly, we can now measure the lattice spacing $a$ and glueball masses $c$ in units of $\sigma$.

\section{CALCULATIONS}

We now implement the procedures described in the first half of the paper for the case of $(2+1)$-dimensional pure large- $N$ gauge theory. The main interest first is to compute glueball masses in physical units. These can be compared with results from established techniques to ascertain whether the transverse lattice theory produces correct results, at least to the level of approximation we use. However, it is worth bearing in mind that, apart from confirming known data, the light-front Hamiltonian approach is uniquely suited to the calculation of hadronic wave functions in a general Lorentz frame, and it is the latter which chiefly motivates us. We will exhibit the structure of these wave functions also. We have also begun analogous calculations in $3+1$ dimensions, which are briefly discussed in the conclusions.

Yang-Mills theory in $2+1$ dimensions is superrenormalizable. Rather than a logarithmic Coulomb potential, a linearly confining interaction appears to be dynamically generated [8]. Therefore it is rather similar to $(3+1)$-dimensional QCD. It provides a useful testbed for nonperturbative calculations, given that two space dimensions can be more efficiently handled numerically than three. The discussion of the first half of the paper can be trivially modified for $2+1$ dimensions (the reader is referred to I for further detail). Our light-front Hamiltonian (26) reduces in $2+1$ dimensions to

$$
\begin{aligned}
P^{-}= & \int d x^{-}-\frac{G^{2}}{4} \operatorname{Tr}\left\{J+\frac{1}{\partial_{-}^{2}} J^{+}\right\}+\mu^{2} \operatorname{Tr}\left\{M M^{\dagger}\right\} \\
& +\frac{\lambda_{1}}{a N} \operatorname{Tr}\left\{M M^{\dagger} M M^{\dagger}\right\}+\frac{\lambda_{2}}{a N} \operatorname{Tr}\left\{M M M^{\dagger} M^{\dagger}\right\} \\
& +\frac{\lambda_{3}}{a N^{2}}\left(\operatorname{Tr}\left\{M M^{\dagger}\right\}\right)^{2}, \\
J^{+}= & i M \stackrel{\leftrightarrow}{\partial}_{-} M^{\dagger}+M^{\dagger} \stackrel{\leftrightarrow}{\partial}_{-} M,
\end{aligned}
$$


TABLE I. The scaling trajectory which minimizes the $\chi^{2}$ test of Lorentz covariance. The couplings $l_{i}, t$, and the overall scale $G^{2} N / \sigma$ are all obtained from the test. $m$ is equivalent to the lattice spacing degree of freedom.

\begin{tabular}{ccccccccc}
\hline \hline$m$ & $l_{1}$ & $l_{2}$ & $l_{3}$ & $t$ & $\frac{G^{2} N}{\sigma}$ & $\chi^{2}$ & $c_{p}$ & $\frac{a^{2} \sigma_{T}^{2}}{G^{2} N}$ \\
\hline 0.134186 & -0.1263 & -0.1178 & 3.5004 & -0.3289 & 5.3375 & 17.5267 & -1.1680 & 0.2834 \\
0.180255 & -0.0772 & -0.2508 & 221.03 & -0.9606 & 5.2220 & 12.1472 & -1.0791 & 0.2699 \\
0.227546 & -0.1606 & -0.1754 & 7.3792 & -1.1712 & 5.4358 & 13.6865 & -1.1836 & 0.3473 \\
0.276458 & -0.1993 & -0.2049 & 6.4575 & -0.9429 & 5.0986 & 10.8355 & -1.1455 & 0.3860 \\
0.327475 & -0.1783 & -0.2133 & 9.4007 & -0.8262 & 4.6138 & 12.6389 & -1.4465 & 0.4592 \\
0.381194 & -0.2032 & -0.1397 & 3.4963 & -1.0544 & 4.5280 & 20.9469 & -1.7720 & 0.5671 \\
0.438370 & -0.1642 & -0.1254 & 2.0687 & -1.2205 & 4.3206 & 21.8354 & -2.2138 & 0.6670 \\
0.500000 & -0.1731 & -0.1284 & 2.3097 & -1.4091 & 4.1691 & 22.6331 & -2.2572 & 0.7603 \\
\hline \hline
\end{tabular}

where now $a G^{2} \rightarrow g_{3}^{2}$ as $a \rightarrow 0$, with $g_{3}^{2}$ the standard $(2+1)$-dimensional Yang-Mills coupling in the continuum limit. ${ }^{4}$ Otherwise, the analysis is unmodified (in particular, the scaling analysis of Sec. VII). The link field $M\left(x^{-}\right)$, of course, now points in one transverse direction only. Under large- $N$ reduction it depends only upon $x^{-}$and is equivalent to two real adjoint matrix fields. We are now ready to diagonalise $P^{-}$in the Fock space of $M(\mathbf{x})$, as a function of $m, l_{1}$, $l_{2}, l_{3}$. Here $G^{2} N$ will be used to set the overall scale for dimensionful physical quantities. It will be determined in terms of a physical scale by measurements of the string tension. The glueball spectrum is classified by $\mid \mathcal{J}^{\mathcal{P}_{1} \mathcal{C}}$, for angular momentum $\mathcal{J}, x^{1}$ reflection symmetry $\mathcal{P}_{1}$, and charge conjugation $\mathcal{C}$. The latter two are exact, while the first can only be estimated approximately on the transverse lattice.

We follow the computational methods established in Ref. [20] for adjoint scalar matter in two dimensions, together with various improvements described in the Appendixes of I and Appendixes $\mathrm{C}$ and $\mathrm{D}$ of this paper. In the following, all results should be assumed extrapolated to the longitudinal continuum limit (this is done before a search for the transverse lattice scaling trajectory is made).

\section{RESULTS}

\section{A. First principles}

To search for a scaling trajectory that restores Lorentz covariance, we applied a $\chi^{2}$ test for certain observables. First, we investigated which observables would be useful in this respect. The natural candidates are the $c_{T} / c_{L}$ ratios deduced from glueball dispersion relations, the Lorentz multiplet degeneracy in the spectrum, and rotational invariance of the heavy-source potential.

\footnotetext{
${ }^{4}$ In I, we did not clearly distinguish between the $a$-dependent transverse lattice coupling $G$ and the continuum coupling. Moreover, the normalization of the continuum gauge coupling in that work was nonstandard. Replace $G^{2}$ by $2 g^{2}$ and $m^{2}$ by $m^{2} / 2$ to convert expressions in this paper to those appearing in I.
}

In order to determine which glueballs it makes sense to include in the $c_{T} / c_{L}$ part of the $\chi^{2}$ test, for each low-lying glueball we determined the $l_{i}$ trajectory such that $c_{T} / c_{L}=1$ for that glueball. In the low-lying spectrum, only the result for the first excited state $0_{*}^{++}$was markedly incompatible with the rest. In fact, it is consistently imaginary. We show below why this artifact is to be expected of our truncation of the effective potential to fourth order. Therefore, excluding this state, we included the $c_{T} / c_{L}$ ratio of the lowest seven glueballs: $0^{++}, 2^{++}, 2^{-+}, 0^{--}, 0_{*}^{--}, 2^{+-}, 2^{--}$. These are the lowest seven glueballs for a broad range of $l_{i}$ at fixed $m$, and also the seven lowest in the ELMC simulations [8].
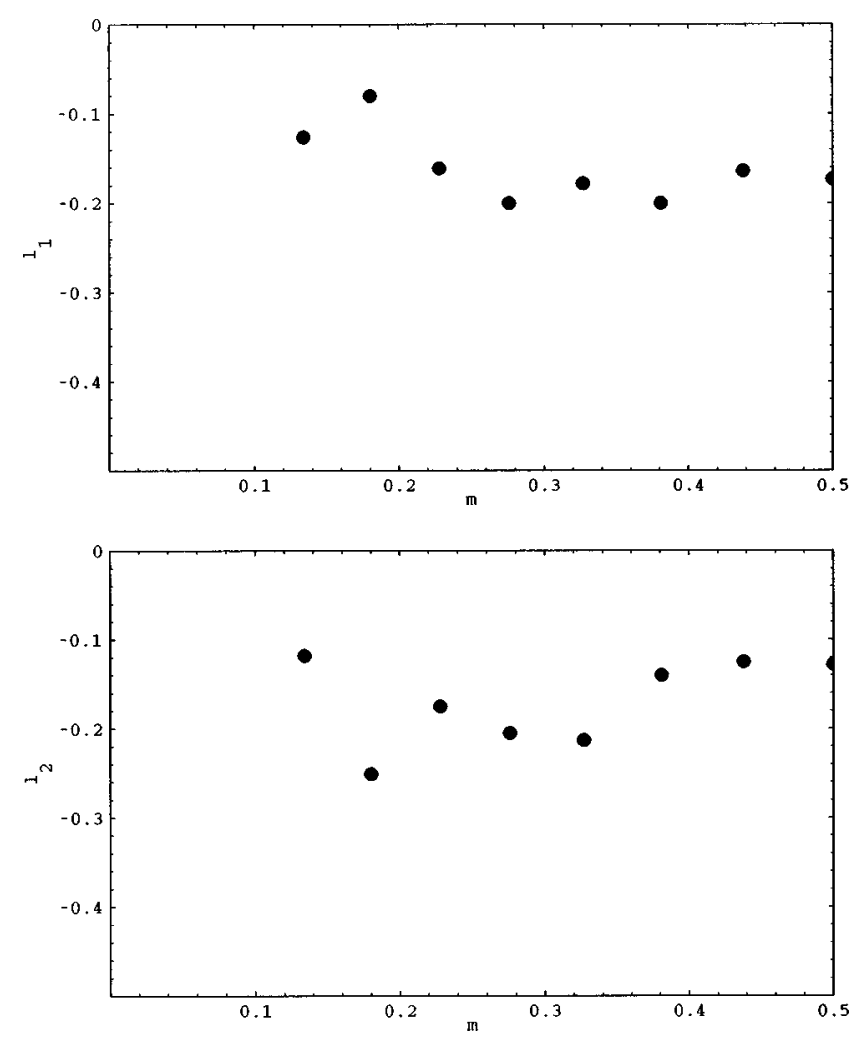

FIG. 3. The trajectory which minimizes the $\chi^{2}$ test of Lorentz covariance; see Table I. 


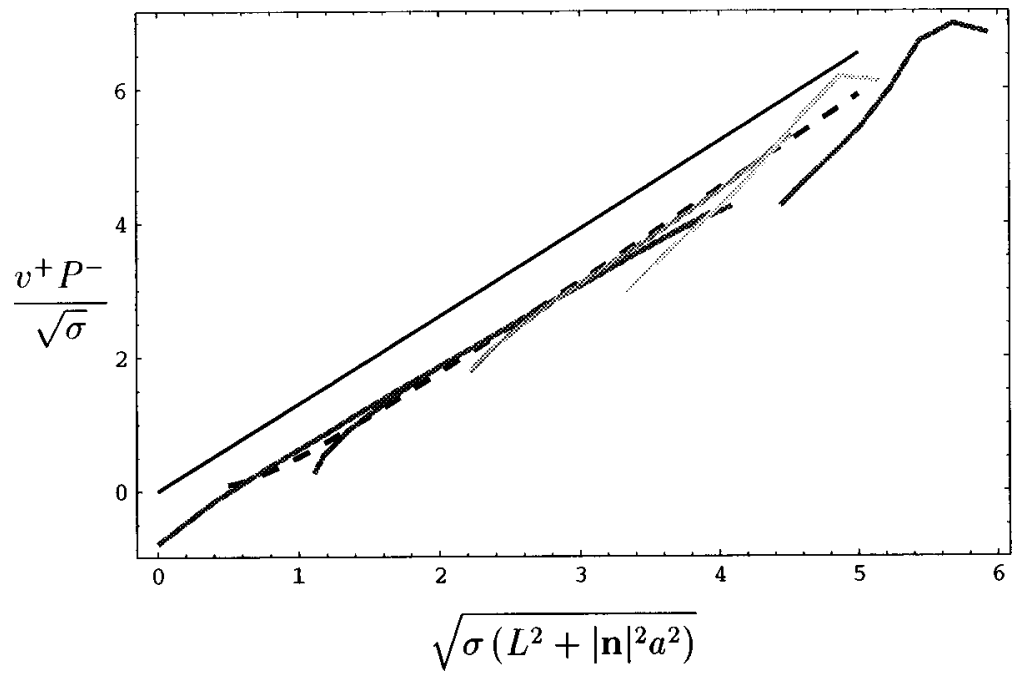

FIG. 4. The shaded lines represent the heavysource potential as a function of separation $\sqrt{\sigma\left(L^{2}+|\mathbf{n}|^{2} a^{2}\right)}$ for $|\mathbf{n}|=0,2,3,4$, where $\mathbf{n}$ is transverse separation. The dashed line is from Eq. (84), and the solid line is the heavy-heavy Coulomb potential from Eq. (60). The eigenvalues were obtained by extrapolating in $K_{\max }, P_{\max }$, and $p$-truncation; the couplings are from the $m$ $=0.180255$ datum of Table I.

Another possible test of Lorentz covariance is the degeneracy of the parity doublets for the $2^{ \pm+}$and the $2^{ \pm-}$. However, we find that there is no point in our parameter space where the $2^{++}$and the $2^{-+}$are degenerate and we do not include this pair in our $\chi^{2}$ test. For the $2^{+-}$and $2^{--}$, we have the opposite problem: the two levels are almost degenerate for a vary wide range of couplings. As a consequence, it does not provide a very useful criterion for finding the correct scaling trajectory (although it was included).

In addition, we demand rotational invariance of the heavy-source potential, based on measurements with zeroand one-link transverse separations. As described in Sec. VII, the longitudinal string tension $\sigma_{L}$ measurement can be used to determine the overall scale $G^{2} N / \sigma$. In fact, this measurement appears very difficult to make accurately, and it proved difficult to establish a scaling trajectory based upon

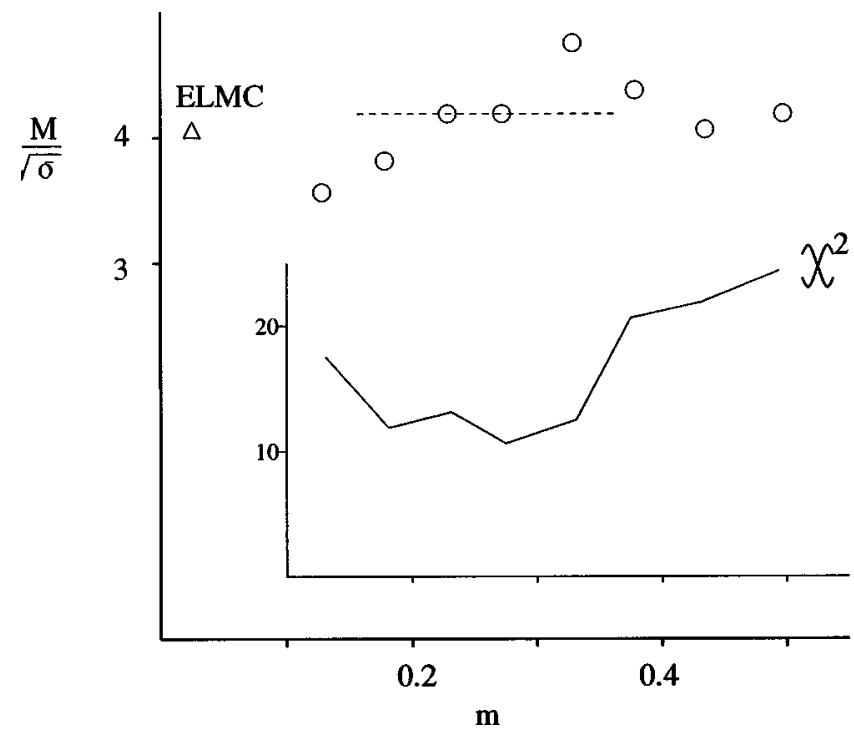

FIG. 5. The $0^{++}$glueball mass and $\chi^{2}$ values along the coupling trajectory of Table I. The size of the data points gives an indication of the error from extrapolation to the longitudinal continuum limit: that is, the dominant error is from scaling violation on the transverse lattice. it. In principle, one might infer the overall scale from demanding consistent dispersion relations of the glueballs alone, without reference to $\sigma_{L}$. However, we found this method fared no better. Instead, we treated $G^{2} N / \sigma$ as free parameter along with the other couplings in the effective potential, but added a term to the $\chi^{2}$ test to minimize deviations from the value one would deduce from $\sigma_{L}$ measurement. In this way the effects of any systematic error in $\sigma_{L}$ are reduced.

The coupling trajectory we find from the resulting $\chi^{2}$ test is shown in Table I and Fig. 3. As previously found in I, the magnitude of $l_{3}$ always comes out much larger than that of $l_{1}$ and $l_{2}$, essentially infinite. Here we are able to confirm that this is required by Lorentz covariance. We also elaborate on this below. Although there are some fluctuations in the coupling trajectory as $m$ as varied, it is nevertheless remarkably close to the trajectory found in I on the basis of a fit to the ELMC spectrum.

In determining the trajectory, we inferred roundness of the heavy-source potential from measurements at transverse separations $|\mathbf{n}|=0$ and 1. If we plot the heavy-source potential for more values of $|\mathbf{n}|$, we observe that rotational invariance of the potential is maintained; see Fig. 4. A potential that is "oval" - that is, rotationally invariant to within an

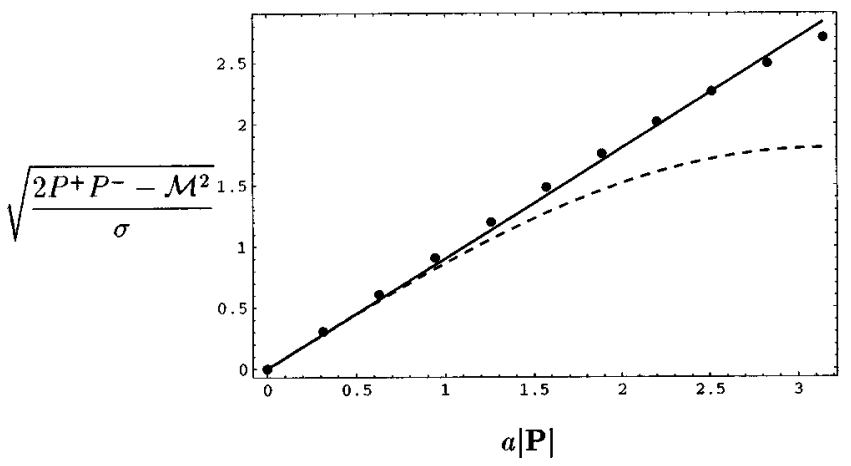

FIG. 6. Dispersion relation for the $0^{++}$up to the edge of the Brillouin zone. The solid line is the relativistically correct dispersion relation, and the dashed line is a lattice dispersion relation. The couplings are from the $m=0.180255$ datum of Table I. 
TABLE II. The glueball masses at $m=0.276$ on the scaling trajectory of Table I at the minimum $\chi^{2}=10.84$.

\begin{tabular}{lcc}
\hline \hline $\mid \mathcal{J}^{\mathcal{P}_{1} C}$ & $M / \sqrt{\sigma}$ & $c_{T} / c_{L}$ \\
\hline $0^{++}$ & 4.16 & 1.10 \\
$0^{--}$ & 4.86 & 0.71 \\
$2^{++}$ & 5.27 & 0.59 \\
$2^{-+}$ & 6.76 & 1.00 \\
$0_{*}^{++}$ & 5.39 & $0.96 i$ \\
$0_{*}^{--}$ & 6.86 & 0.87 \\
$2^{+-}$ & 7.32 & 0.39 \\
$2^{--}$ & 7.64 & 0.38 \\
\hline \hline
\end{tabular}

overall $\sigma_{T} / \sigma_{L}$-seems to be generic for the transverse lattice [10]. Consequently, the crucial test is whether one obtains consistent longitudinal versus transverse scales $\sigma_{T}=\sigma_{L}$ $=\sigma$.

Examining the glueball masses on this trajectory more closely, we find results for the $0^{++}$ground-state mass shown in Fig. 5. It is to be anticipated that the $\chi^{2}$ becomes poor at large and small $m$. At large $m$, corresponding to large $a$ (see Fig. 8), the truncated effective potential cannot cope with the transverse discretization errors. At small $m$, the wave functions are most sensitive to the the small- $k^{+}$region and, hence, the longitudinal discretization errors. It is also the edge of the color-dielectric regime. The $0^{++} c_{T} / c_{L}$ ratio is $1 \pm 0.15$ all along the coupling trajectory and the mass scales to some degree. Based on this, with a generous error for scaling violation, we estimate $M_{0^{++}} / \sqrt{\sigma}=4.05 \pm 0.3$, in good agreement with the ELMC value ${ }^{5} 4.08(7)$ (stat).

At the best $\chi^{2}$, we list the masses and $c_{T} / c_{L}$ ratios of the other low-lying glueballs in Table II. The level ordering of the seven glueballs is in agreement with the ELMC result, and the numerical values are generally not far off. We note, however, that, with one exception, we cannot get the higher glueballs, along with the string tension and $0^{++}$, to be especially rotationally invariant for our approximation to the effective potential. It is perhaps not coincidental that both the masses and $c_{T} / c_{L}$ ratios of the higher glueballs come out consistently too low. The one exception, $2^{-+}$, is in good agreement with the ELMC mass 6.89(21)(stat).

In Figs. 6 and 7, we plot the dispersion relation for the $0^{++}$and $0^{--}$for a point on the scaling trajectory. This is a consistency check, to show whether optimizing the slope at $|\mathbf{P}|=0$ leads to covariant behavior throughout the Brillouin zone. Behavior in the figures is generic: the dispersion of the $0^{++}$is extremely straight throughout the scaling trajectory and the dispersion of the $0^{--}$, along with the other excited states, tends to follow a lattice dispersion $\propto \sqrt{1-\cos (a|\mathbf{P}|)}$ more closely.

\footnotetext{
${ }^{5}$ The only other accurate value we know of comes from an SU(3) equal-time Hamiltonian lattice calculation [28]. However, apart from $1 / N^{2}$ corrections, it cannot be compared directly with our result since the authors do not measure the string tension, but give results in terms of a gauge coupling.
}

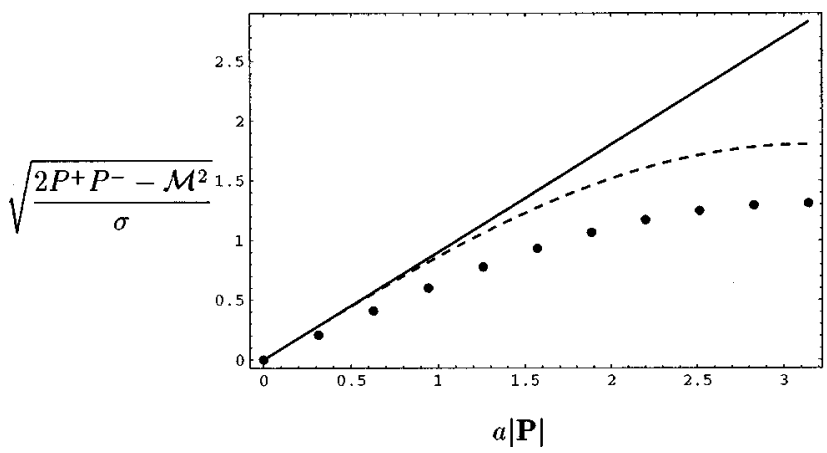

FIG. 7. Dispersion relation for the $0^{--}$up to the edge of the Brillouin zone. The solid line is the relativistically correct dispersion relation, and the dashed line is a lattice dispersion relation (normalized to the same slope at $|\mathbf{P}|=0$ ). The $0^{--}$typically has a poor slope at $|\mathbf{P}|=0$ and follows a lattice dispersion more closely. The couplings are from the $m=0.180255$ datum of Table I.

We can also deduce the transverse lattice spacing $a$ in physical units $\sqrt{\sigma}$, as one moves along the trajectory of Table I (see Fig. 8). This confirms that the lattice spacing gradually decreases with $m$ and is always physically quite large in the color-dielectric regime $m^{2}>0$. Further information can be gleaned from the glueball wave functions, which of course come for free in a Hamiltonian calculation. In Table III and Fig. 9, we exhibit the transverse and longitudinal structure of the glueballs at a representative point in coupling space. We see a characteristic rise of the $G_{d}(x)$ distribution at small $x$, due to higher Fock states. However, one can show analytically that such distributions must vanish at $x=0$ for two-dimensional gauge theories [29] (without Yukawa interactions) and, therefore, our color-dielectric regime $m^{2}>0$ in particular.

The coupling $l_{3}$, according to the test for Lorentz covariance, is much larger than other couplings, which might appear unnatural at first sight. However, the corresponding operator is also distinguished by the fact that at large $N$ it couples only to link-antilink Fock states (it "pinches" the flux tube swept out light-front time $\left.x^{+}\right)$. More precisely, if the link-antilink wave function is $f\left(k^{+}, P^{+}-k^{+}\right)$, the $l^{3}$ operator has expectation value

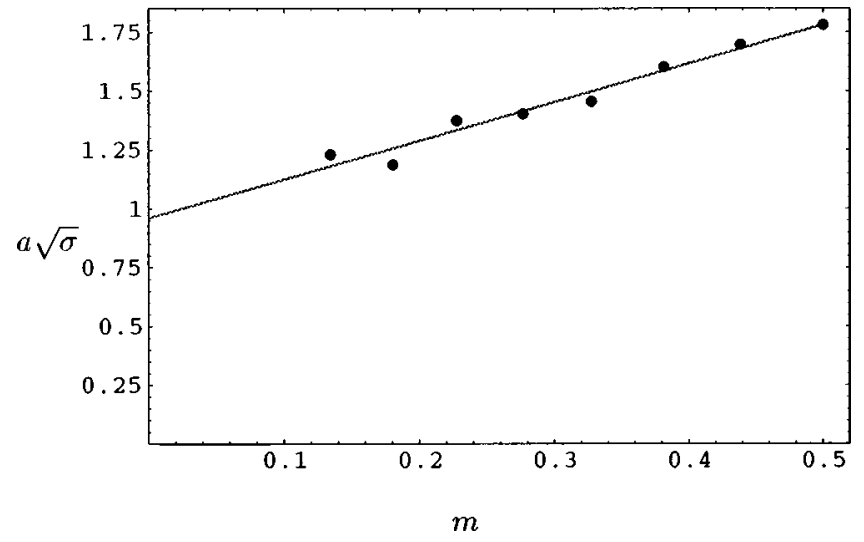

FIG. 8. Variation of the lattice spacing $a$ vs $m$ along the scaling trajectory of Table I. Also shown is a fit to $0.96+1.64 m$. 
TABLE III. The transverse structure of each glueball for $K$ $=8, p \leqslant 6$, at $m=0.134, l_{1}=-0.153, l_{2}=-0.105, l_{3}=100$. Each column shows the probability for a glueball to contain a loop of given transverse shape. Information on the relative phases at different shapes can similarly be obtained from the wave functions.

\begin{tabular}{|c|c|c|c|c|c|c|}
\hline$|\mathcal{J}|^{\mathcal{P}_{1} \mathcal{C}}$ & $\begin{array}{l}x \\
\int_{x}^{x}\end{array}$ & $\begin{array}{l}x \\
\int^{*}\end{array}$ & $\begin{array}{l}{ }^{x} \\
\times\end{array}$ & $\int_{x}^{\infty}$ & $\sqrt{x}$ & $\begin{array}{c}x \\
x \\
x\end{array}$ \\
\hline $0^{++}$ & 0.007 & 0.184 & 0.676 & 0.002 & 0.022 & 0.108 \\
\hline $0_{*}^{++}$ & 0 & 0.716 & 0.189 & 0.009 & 0.027 & 0.059 \\
\hline $0^{--}$ & 0.889 & 0.089 & 0.018 & 0.001 & 0.002 & 0.002 \\
\hline $0_{*}^{--}$ & 0.069 & 0.68 & 0.118 & 0.023 & 0.053 & 0.056 \\
\hline $2^{++}$ & 0.76 & 0.061 & 0.14 & 0.001 & 0.007 & 0.03 \\
\hline $2^{-+}$ & 0 & 0.871 & 0.002 & 0.005 & 0.061 & 0.061 \\
\hline
\end{tabular}

$$
\left\langle\left(\operatorname{Tr}\left\{M M^{\dagger}\right\}\right)^{2}\right\rangle \propto\left(\int_{0}^{P^{+}} d k^{+} \frac{f\left(k^{+}, P^{+}-k^{+}\right)}{\sqrt{k^{+}\left(P^{+}-k^{+}\right)}}\right)^{2}
$$

A complete set of link-antilink wave functions $f$ resulting from the longitudinal Coulomb interaction can be labeled by the number of zeros of $f[2]$. The energy increases with the number of zeros, and one might naively expect the $0^{++}$ glueball to therefore be predominantly composed of a constant two-link wave function. However, this wave function and only this wave function makes a significant contribution to Eq. (45). A large $l_{3}$ therefore removes it from all lowlying glueballs, as is evidenced from Table III and Fig. 9. An intuitive explanation for this follows from the fact that a wave function constant in $k^{+}$implies tight binding in the longitudinal direction. On the other hand, the link-antilink state has width $a$ in the transverse direction. From Fig. 8 we know this to be physically quite large (of order the glueball width). Therefore it is perhaps not surprising that the Lorentz covariance conditions reject such an asymmetrical Fock wave function in low-lying glueballs. If this conclusion carries over to $3+1$ dimensions, it will result in a quite different picture from the one suggested in Ref. [3] (which did not include a $l_{3}$ coupling).

The absence of the link-antilink state means that the $0^{++}$ ground-state glueball is predominantly formed from a linear combination of the four-link operators $\mathcal{O}_{1}=\operatorname{Tr}\left\{M^{\dagger} M M^{\dagger} M\right\}$ and $\mathcal{O}_{2}=\operatorname{Tr}\left\{M M M^{\dagger} M^{\dagger}\right\}$. In fact, we find that the $\left(0^{++}, 0_{*}^{++}\right)$system is to a good approximation described by the symmetric and antisymmetric combinations $\left(\alpha \mathcal{O}_{1}\right.$
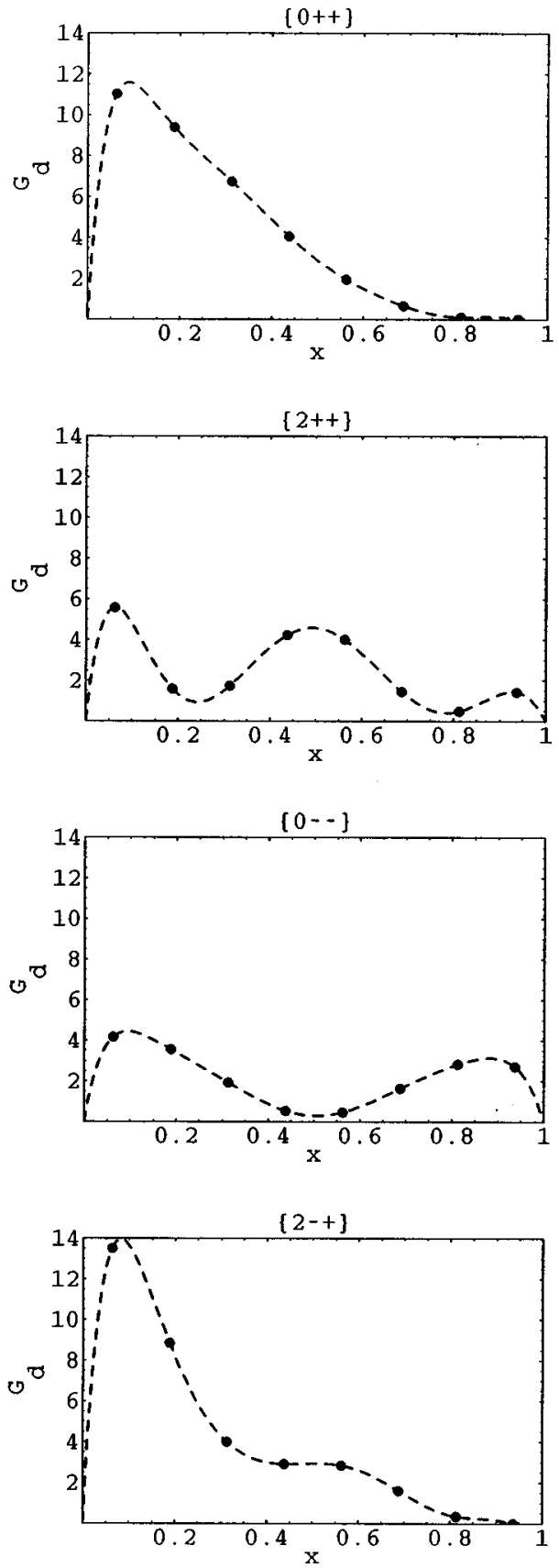

FIG. 9. The structure functions $G_{d}(x)$ for $K=8, p \leqslant 6$, at $m$ $=0.134, l_{1}=-0.153, l_{2}=-0.105, l_{3}=100$. This exhibits the longitudinal structure of each glueball.

$\left.+\beta \mathcal{O}_{2}, \alpha \mathcal{O}_{1}-\beta \mathcal{O}_{2}\right)$. The operator $\mathcal{O}_{2}$ couples to $l_{2}<0$ in the Hamiltonian and is the most significant hopping term on the transverse lattice. As a result, we find the symmetric combination lower in energy and with correct relativistic dispersion $c_{T}^{2}>0$. It then follows unavoidably that the higherenergy antisymmetric combination will have an incorrect dispersion $c_{T}^{2}<0$. Since we tentatively identified the antisymmetric combination with the $0_{*}^{++}$, this explains why we always have a problem with this state. In fact, it is an almost purely lattice artifact at present. It is likely that the effective potential must be extended to six-link operators before this problem can be resolved. 

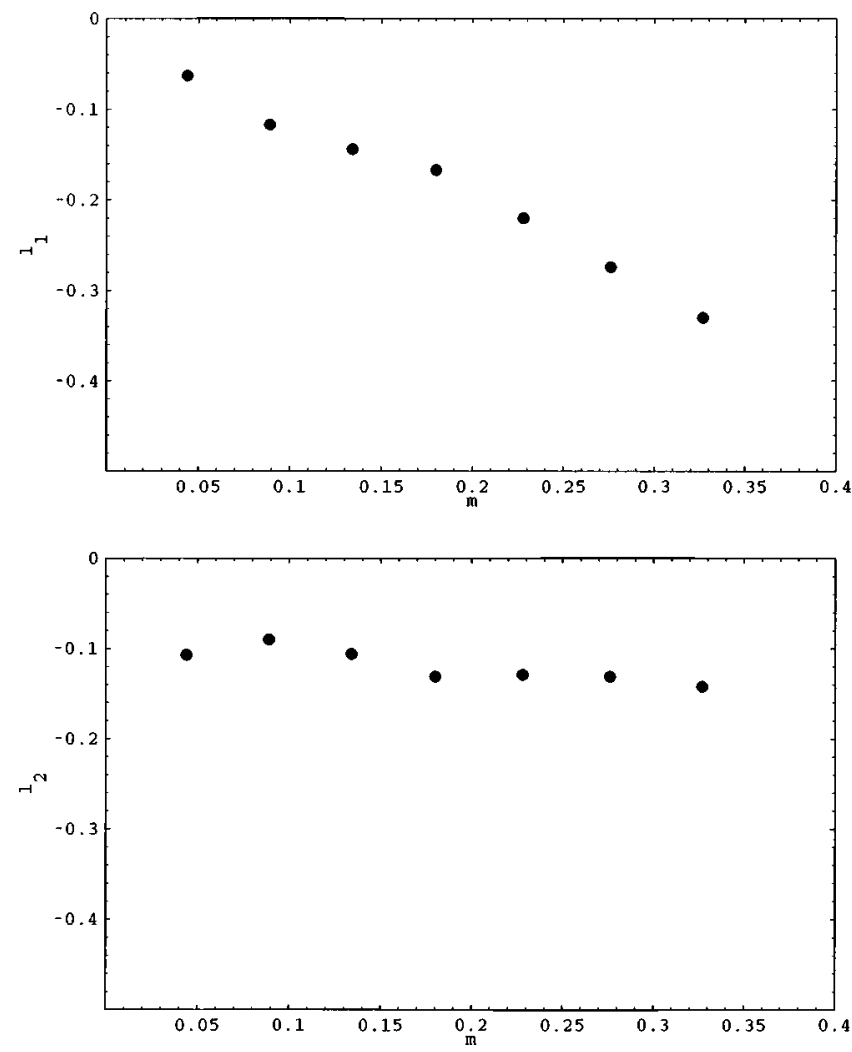

FIG. 10. The trajectory which minimizes the $\chi^{2}$ test of Lorentz invariance involving only glueball dispersion formulas.

\section{B. Improving higher glueballs}

We have found in the previous section that, determining the glueball masses in terms of a scale set by a rotationally invariant string tension, we can obtain a reasonable ground state $0^{++}$, but cannot obtain Lorentz-covariant higher glueballs in general. We attribute this incompatibility to our truncation of the effective potential. It is natural to ask if one could find a coupling trajectory which improves the Lorentz covariance of higher glueballs at the expense of rotational invariance of the string tension. In particular, the longitudinal string tension is by far the most difficult measurement we make and a potential source of systematic errors. To this end, we searched for such a trajectory, using the ELMC value of $M_{0++} / \sqrt{\sigma}$ as a phenomenological input to set the overall scale. $\sigma$ was identified with $\sigma_{T}$ in this case. Although no longer quite a first-principles calculation, the results are sufficiently impressive, we feel, to display here.

Using a $\chi^{2}$ criterion which includes only the $c_{T} / c_{L}$ ratio of the $0^{++}$(whose mass is now fixed) and the $0^{--}$, we found the trajectory displayed in Fig. 10. In fact, along this trajectory, not only are these two glueballs rotationally invariant, but a number of others also have a good $c_{T} / c_{L}$ ratio. The trajectory is close to the previous one, but rather more stable. The scaling behavior of glueball masses, shown in Fig. 11, is also more stable. At the point of minimum $\chi^{2}$ on this trajectory, we find the spectrum displayed in Fig. 12. In particular, it is worth noting that those glueballs whose $c_{T} / c_{L}$ ratio is close to 1 have predicted masses in agreement with the ELMC result. This encourages us that adding fur- ther couplings to the effective potential to improve the Lorentz covariance of higher glueballs (from first principles) should bring their masses into line also.

\section{CONCLUSIONS}

We have constructed an effective transverse lattice lightfront Hamiltonian of pure gauge theory, extending the earlier works $[1,2,3]$, and outlined a first-principles procedure for solving the eigenvalue problem. Not only does this formulation of gauge theory possess the usual appealing features associated with light-front quantization-trivial vacuum, boost invariance, parton dynamics-but it also efficiently deals with the common problems found in this approachzero modes, renormalization, noncompactness, and gauge invariance. It does this at the price of introducing an effective potential which must be tuned by demanding Lorentz covariance of eigenstates of the light-front Hamiltonian at large transverse lattice spacing. Further remarkable simplifications occur in the large- $N$ limit. Only a $(1+1)$-dimensional theory of connected Wilson loops needs to be solved. Equivalently, one solves a $(1+1)$-dimensional continuum gauge theory coupled to complex adjoint matter fields which form freely propagating color strings.

We successfully tested the ideas by performing comprehensive calculations in $2+1$ dimensions at large $N$, using an improvement scheme to extrapolate to the longitudinal continuum limit. We found agreement with conventional Euclidean lattice simulations [8], in those observables for which Lorentz covariance was attained. Furthermore, we could go beyond the path integral approach by obtaining the frameinvariant glueball wave functions. Given that the quantization, regulators, elementary fields, and gauge fixing are different from those of Ref. [8], the agreement is quite impressive. It nonperturbatively validates both the lattice color-dielectric formulation and the light-front Hamiltonian quantization of non-Abelian gauge theory at the quantitative level. This is therefore an important step in the application of light-front quantization to hadronic physics in general.

The obvious extension of large- $N$ pure glue calculations to $3+1$ dimensions has already begun, and initial results are encouraging. For couplings in the light-front Hamiltonian (26) similar to those found for the $2+1$ problem, we find $0^{++}, 2^{++}$, and $1^{+-}$multiplets as the lightest glueballs (only some of the components of these multiplets were found in the original work [3]). A search of the full parameter space for a scaling trajectory will yield estimates of the masses and wave functions. Given that $1 / N^{2}$ corrections are expected to be smaller than other systematic errors, the masses can be compared with various experimental glueball candidates, providing a useful alternative to the conventional ELMC estimates.

\section{ACKNOWLEDGMENTS}

S.D. thanks Professor F. Lenz (Erlangen) for hospitality during part of the work and M. Teper for many helpful interactions. The work of S.D. was supported by the Particle Physics and Astronomy Research Council (U.K.) and CERN. 

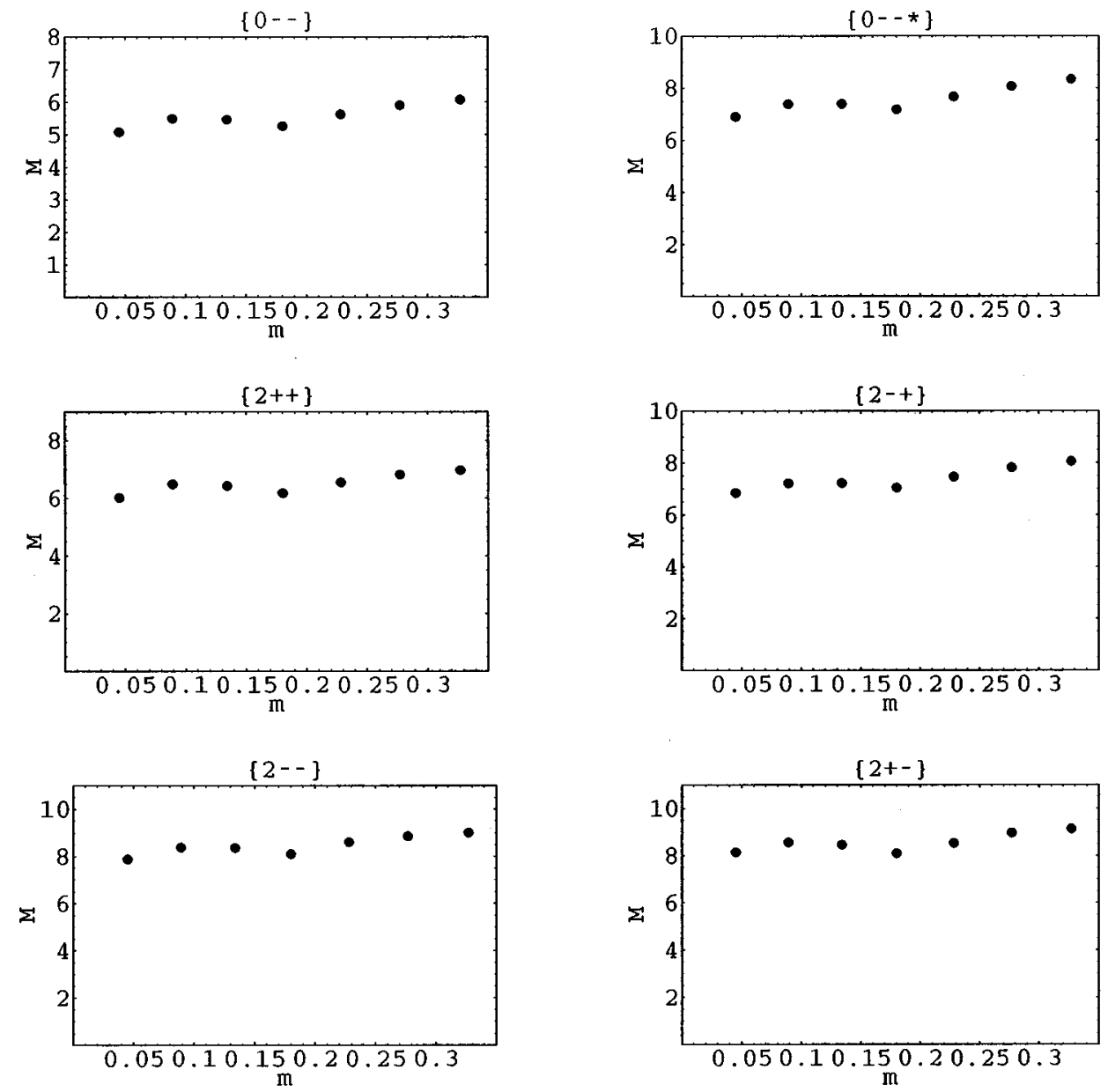

FIG. 11. Scaling of the higher glueball masses $\mathcal{M}$ in units of $\sqrt{\sigma}$, as $m$ is varied along the scaling trajectory of Fig. 10. This gives an estimate of systematic errors for each glueball from truncation of $V$.

\section{APPENDIX A: HEAVY-SOURCE POTENTIAL}

Here we describe our procedure for measuring the potential between two heavy sources. For convenience, we will start with a heavy scalar field $\phi\left(x^{+}, x^{-}, \mathbf{x}\right)$ of mass $\rho$ in the fundamental representation of the color group (one can just as well start with the Dirac equation and take the heavy limit; the result is the same). In addition to the pure glue "linklink" interactions, the most general action to fourth order in the fields is

$$
\begin{array}{r}
\sum_{\mathbf{x}} \int d x^{-} d x^{+}\left(D_{\alpha} \phi\right)^{\dagger} D^{\alpha} \phi-\rho^{2} \phi^{\dagger} \phi \\
-\frac{\rho \tau}{a^{D-3} N} \phi^{\dagger}\left(M_{r}^{\dagger} M_{r}+M_{r} M_{r}^{\dagger}\right) \phi,
\end{array}
$$

where

$$
D_{\alpha} \phi^{\dagger}=\partial_{\alpha} \phi^{\dagger}-i \phi^{\dagger} A_{\alpha}
$$

The heavy field contributes to the gauge current $J^{\alpha}$ in Eq. (11):

$$
J_{\text {heavy }}^{\alpha}=-i\left(D^{\alpha} \phi\right) \phi^{\dagger}+i \phi\left(D^{\alpha} \phi\right)^{\dagger} \text {; }
$$

note that $\phi \phi^{\dagger}$ is an $N \times N$ color matrix. Since we are interested in the $\rho \rightarrow \infty$ limit, we have not included any hopping terms like $\phi^{\dagger}(\mathbf{x}) M_{r}(\mathbf{x}) \phi(\mathbf{x}+a \hat{r})$ and $\phi(\mathbf{x})$ is pinned to site $\mathbf{x}$ of the lattice. Also, we have not included any "pinching terms" like $\phi^{\dagger} \phi \phi^{\dagger} \phi$, which, for large $N$, act only on states consisting of two $\phi$ 's.

Now for some kinematics: Let $P_{\text {full }}^{\alpha}$ represent the full two-momentum of a system containing $h$ heavy particles. It is convenient to split the full momentum into a "heavy" part plus a "residual" part $P^{\alpha}$, 


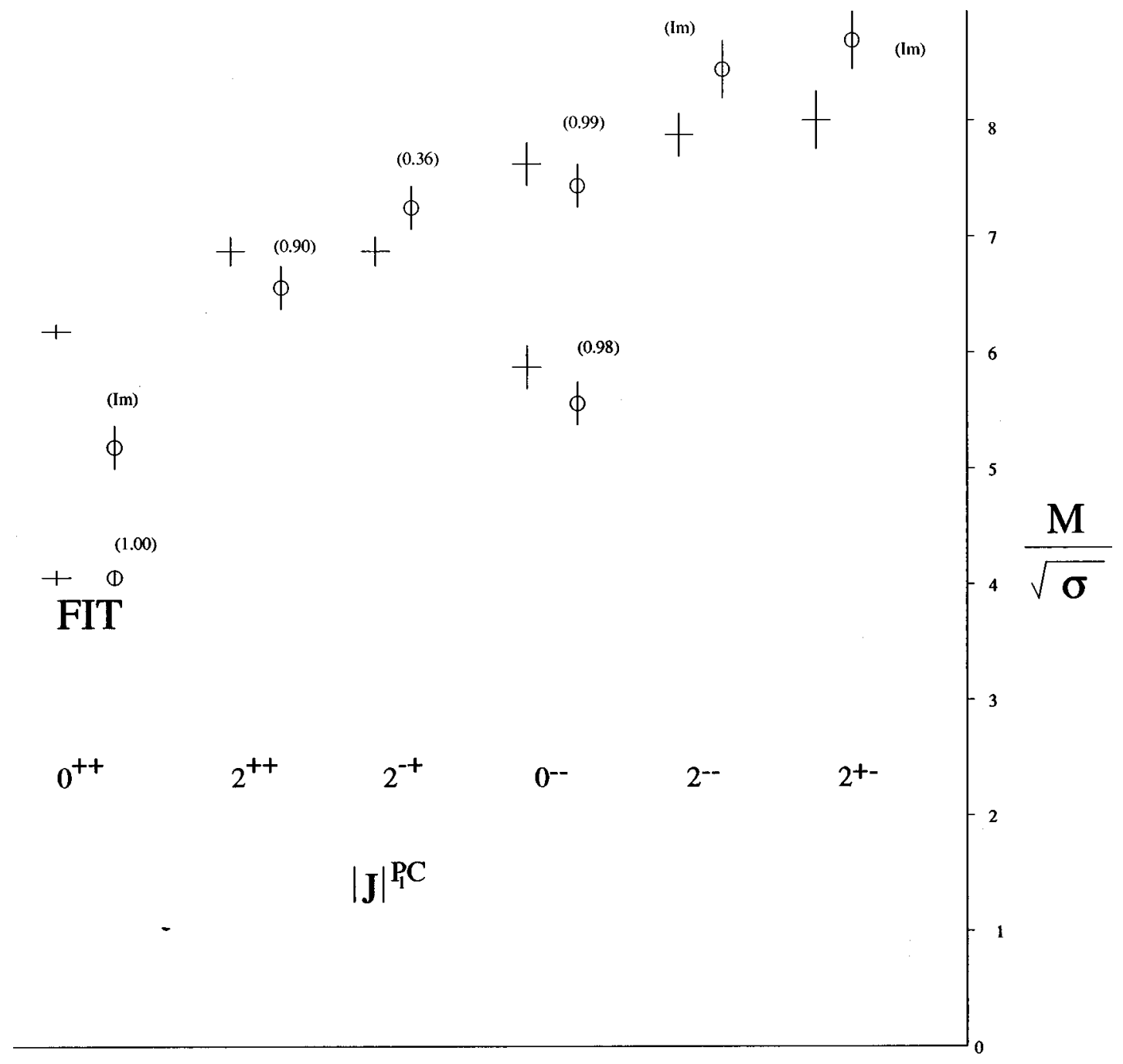

FIG. 12. The low-lying (2+1)-dimensional large- $N$ glueball spectrum obtained on the transverse lattice $(\bigcirc)$. Also shown for comparison, the ELMC simulations of Teper $(+)$, extrapolated to $N=\infty$ by a fit to $A+B / N^{2}$, with statistical errors [8]. The value of $M_{0++} / \sqrt{\sigma}$ was fit to the ELMC result, to set the scale. The other masses result from requiring rotationally invariant dispersion, quantified by the speed of light ratio $c_{T} / c_{L}$ deduced from each glueball (in brackets). The estimated error from extrapolation in $K$ and $p$ is shown but an estimate of systematic error from truncation of $V$ is not shown (see Fig. 11).

where $v^{\alpha}$ is the covariant velocity of the heavy quarks, $v^{\alpha} v_{\alpha}=1$. The full invariant mass squared $(\mathbf{P}=0)$ is

$$
\mathcal{M}^{2}=2 P_{\text {full }}^{+} P_{\text {full }}^{-}=(h \rho)^{2}+2 h \rho v^{+} P^{-}+2 P^{+}\left(P^{-}+h \rho v^{-}\right) .
$$

Our choice of $v^{+}$is arbitrary, and it is convenient to choose it such that $P^{+}=0$. Consequently, $v^{+} P^{-}$is just the shift of the full invariant mass $\mathcal{M}$ due to the interactions:

$$
\mathcal{M}=h \rho+v^{+} P^{-}+O(1 / \rho) .
$$

Thus $v^{+} P^{-}$is the usual energy associated with the heavyquark potential; this will be our Hamiltonian.
We define creation and annihilation operators associated with the heavy field:

$$
\begin{aligned}
\phi= & \frac{1}{\sqrt{4 \pi}} \int_{-\infty}^{\infty} \frac{d k}{\sqrt{\rho v^{+}+k}} \\
& \times\left[b(k) e^{-i v_{\alpha} x^{\alpha} \rho-i k x^{-}}+d^{\dagger}(k) e^{i v_{\alpha} x^{\alpha} \rho+i k x^{-}}\right] .
\end{aligned}
$$

The creation and annihilation operators $b(k)$ and $d(k)$ have the usual commutation relations. The $e^{i \rho v_{\alpha} x^{\alpha}}$ term removes an overall momentum $\rho v^{\alpha}$ from the two-momentum. The contribution of the heavy-particle interactions to the "residual" momenta $P^{\alpha}$ in Eqs. (12) and (13) are, to leading order in large $\rho$ and $N$, 


$$
\begin{aligned}
P_{\text {heavy }}^{+}= & \int_{-\infty}^{\infty} d k k\left[b^{\dagger}(k) b(k)+: d(k) d^{\dagger}(k):\right] \\
P_{\text {heavy }}^{-}= & -\int_{-\infty}^{\infty} d k \frac{k}{2 v^{+^{2}}}\left[b^{\dagger}(k) b(k)+: d(k) d^{\dagger}(k):\right] \\
& -\frac{G^{2}}{4 \pi} \int d k_{1} d k_{2} d k_{3} d k_{4} \frac{\delta\left(k_{1}+k_{2}-k_{3}-k_{4}\right)}{\left(k_{1}-k_{3}\right)^{2}} b^{\dagger}\left(k_{1}\right) d^{\dagger}\left(k_{2}\right) d\left(k_{4}\right) b\left(k_{3}\right) \\
& +\frac{1}{8 \pi} \int \frac{d k_{1} d k_{2} d k_{3} d k_{4}}{\sqrt{k_{2} k_{3}}}\left[\delta\left(k_{1}+k_{2}-k_{3}-k_{4}\right) \mathcal{H}_{2 \rightarrow 2}+\delta\left(k_{1}-k_{2}-k_{3}-k_{4}\right)\left(\mathcal{H}_{1 \rightarrow 3}+\mathcal{H}_{3 \rightarrow 1}\right)\right]
\end{aligned}
$$

with

$$
\begin{aligned}
& \mathcal{H}_{2 \rightarrow 2}=\left(-\frac{G^{2}\left(k_{2}+k_{3}\right)}{\left(k_{2}-k_{3}\right)^{2}}+\frac{\tau}{a^{D-3} N}\right)\left[: d\left(k_{1}\right) a_{\lambda}\left(k_{2}\right) a_{\lambda}^{\dagger}\left(k_{3}\right) d^{\dagger}\left(k_{4}\right):+b^{\dagger}\left(k_{1}\right) a_{\lambda}^{\dagger}\left(k_{2}\right) a_{\lambda}\left(k_{3}\right) b\left(k_{4}\right)\right], \\
& \mathcal{H}_{1 \rightarrow 3}=\left(\frac{G^{2}\left(k_{2}-k_{3}\right)}{\left(k_{2}+k_{3}\right)^{2}}+\frac{\tau}{a^{D-3} N}\right)\left[: d\left(k_{1}\right) a_{\lambda}^{\dagger}\left(k_{2}\right) a_{-\lambda}^{\dagger}\left(k_{3}\right) d^{\dagger}\left(k_{4}\right):+b^{\dagger}\left(k_{4}\right) a_{\lambda}^{\dagger}\left(k_{3}\right) a_{-\lambda}^{\dagger}\left(k_{2}\right) b\left(k_{1}\right)\right], \\
& \mathcal{H}_{3 \rightarrow 1}=\mathcal{H}_{1 \rightarrow 3}^{\dagger} .
\end{aligned}
$$

As an example, let us construct a state with two heavy sources with rest frame separation $L$ in $x^{D-1}$ (this corresponds to an $x^{-}$separation of $\left.2 v^{-} L\right)$ :

$$
\begin{aligned}
|2\rangle & =\frac{2 \rho v^{+}}{\sqrt{N}} \int d x^{-} \phi^{\dagger}\left(x^{+}, x^{-}+L v^{-}\right) \phi\left(x^{+}, x^{-}-L v^{-}\right)|0\rangle e^{-2 i \rho v_{\alpha} x^{\alpha}} \\
& =\frac{1}{\sqrt{N}} \int_{-\infty}^{\infty} d k b^{\dagger}(k) d^{\dagger}(-k) e^{2 i L v^{-} k} .
\end{aligned}
$$

The associated energy shift is

$$
\frac{\left\langle 2\left|v^{+} P^{-}\right| 2\right\rangle}{\langle 2 \mid 2\rangle}=\frac{G^{2} N|L|}{4}
$$

Thus, as expected, we have linear confinement with string tension of $1 / 4$ in units of $G^{2} N$.

Now let us investigate the bound-state equation for the general case. A state containing two heavy sources with rest frame separation $L$ has the form (suppressing orientation indices $\lambda, \rho$ )

$$
|\psi\rangle=\sum_{p} \frac{1}{\sqrt{N^{p+1}}} \int_{0}^{P_{\text {link }}^{+}<v^{+} \sqrt{G^{2} N} P_{\max }} d k_{1} \cdots d k_{p} \psi\left(k_{1}, \ldots, k_{p}\right) \int_{-\infty}^{\infty} d l e^{2 i L l v^{-}} b^{\dagger}(l-K / 2) a^{\dagger}\left(k_{1}\right) \cdots a^{\dagger}\left(k_{p}\right) d^{\dagger}(-l-K / 2),
$$

where $P_{\text {link }}^{+}=\Sigma_{i} k_{i}$ is the total longitudinal momenta of the link fields. The associated bound-state equation has the form 


$$
\begin{aligned}
& P^{-} v^{+} \psi\left(k_{1}, \ldots, k_{p}\right)=\frac{P_{\text {link }}^{+}}{2 v^{+}} \psi\left(k_{1}, \ldots\right)-\frac{G^{2} N v^{+}}{8 \pi} \int \frac{d k^{\prime}}{\sqrt{k_{1} k^{\prime}}} \frac{k_{1}+k^{\prime}}{\left(k_{1}-k^{\prime}\right)^{2}} \psi\left(k^{\prime}, k_{2}, \ldots\right) e^{i L v^{-}\left(k_{1}-k^{\prime}\right)} \\
& +\frac{\tau}{8 \pi a^{D-3}} \int \frac{d k^{\prime}}{\sqrt{k_{1} k^{\prime}}} \psi\left(k^{\prime}, k_{2}, \ldots\right) e^{i L v^{-}\left(k_{1}-k^{\prime}\right)} \\
& +\frac{G^{2} N v^{+}}{8 \pi} \int \frac{d k_{1}^{\prime} d k_{2}^{\prime}}{\sqrt{k_{1}^{\prime} k_{2}^{\prime}}} \frac{k_{2}^{\prime}-k_{1}^{\prime}}{\left(k_{1}^{\prime}+k_{2}^{\prime}\right)^{2}} \psi\left(k_{1}^{\prime}, k_{2}^{\prime}, k_{1}, \ldots\right) e^{-i L v^{-}\left(k_{1}^{\prime}+k_{2}^{\prime}\right)} \\
& +\frac{\tau}{8 \pi a^{D-3}} \int \frac{d k_{1}^{\prime} d k_{2}^{\prime}}{\sqrt{k_{1}^{\prime} k_{2}^{\prime}}} \psi\left(k_{1}^{\prime}, k_{2}^{\prime}, k_{1}, \ldots\right) e^{-i L v^{-}\left(k_{1}^{\prime}+k_{2}^{\prime}\right)}+\frac{G^{2} N v^{+}}{8 \pi} \frac{k_{2}-k_{1}}{\sqrt{k_{1} k_{2}}\left(k_{1}+k_{2}\right)^{2}} \psi\left(k_{3}, \ldots\right) e^{i L v^{-}\left(k_{1}+k_{2}\right)} \\
& +\frac{\tau}{8 \pi a^{D-3}} \frac{1}{\sqrt{k_{1} k_{2}}} \psi\left(k_{3}, \ldots\right) e^{i L v^{-}\left(k_{1}+k_{2}\right)} \\
& +v^{+}[\text {"link-link" interactions from Eq. (26)]+"source-link" interactions on the other }
\end{aligned}
$$$$
\text { end of the string (complex conjugate of above). }
$$

Note that the first term scales with momentum as $\left(P_{\text {link }}^{+}\right)^{1}$, the $\tau$ terms scale as $\left(P_{\text {link }}^{+}\right)^{0}$, and all other terms scale as $\left(P_{\text {link }}^{+}\right)^{-1}$. Thus the longitudinal momentum scale is determined as a balance between the first term and the remaining terms of the Hamiltonian. As opposed to the closed string, an instantaneous-annihilation process via $A_{+}$can act in the $p$ $=2$ link sector and the "pinching', terms (for example, $\lambda_{3}$ ) do not come into play; otherwise, there are no changes in the "link-link" interactions from the closed string case.

There is a variety of possible cutoffs for $k_{i}$ that one might choose. The cutoff used here $P_{\text {link }}^{+}<v^{+} \sqrt{G^{2} N} P_{\max }$ is convenient because the "link-link" interactions, which act on the interior of the string, conserve $P_{\text {link }}^{+}$. Holding the other momenta constant and ignoring particle-number-changing interactions, we see that $k_{1}$ has the limiting behaviors ${ }^{6}$

$$
\lim _{k_{1} \rightarrow 0} \psi\left(k_{1}, \ldots\right) \propto k_{1}^{\beta}, \quad 0<\beta<1 / 2, \quad \frac{\mu^{2}}{G^{2} N}=\beta \tan (\pi \beta),
$$

$$
\lim _{k_{1} \rightarrow \infty} \psi\left(k_{1}, \ldots\right) \propto\left(\frac{\tau}{a^{D-3} k_{1}^{3 / 2}}-\frac{G^{2} N v^{+}}{k_{1}^{5 / 2}}\right) e^{i L v^{-} k_{1}} .
$$

The first relation is exactly the same "end-point behavior" that occurs for particles in the interior of the string. The second relation (A19) implies that the eigenvalues of $v^{+} P^{-}$ converge as $1 /\left(P_{\max }\right)^{5}$ for $\tau=0$ and $1 /\left(P_{\max }\right)^{3}$ for $\tau$ nonzero.

We solve the bound-state equation (A17) by rescaling and discretizing the longitudinal momenta

\footnotetext{
${ }^{6}$ The $\beta$ here is conventional notation for the longitudinal end-point behavior, not to be confused with the plaquette coupling in Eq. (26).
}

$$
k_{1} \Rightarrow \frac{v^{+} \sqrt{G^{2} N} P_{\max }}{K_{\max }} \widetilde{k}_{i}, \quad \widetilde{k}_{i} \in\{1 / 2,3 / 2, \ldots\},
$$

where $K_{\max } \geqslant K_{\text {link }}=\sum_{i} \widetilde{k}_{i}$ is the maximum total discretized momentum. The ratio $P_{\max } / K_{\max }$ parametrizes the coarseness of the discretization. The discretized bound-state equation, expressed in terms of the dimensionless parameters $P_{\text {max }}$ and $L \sqrt{G^{2} N}$, is linear in the couplings

$$
\sqrt{G^{2} N}, \quad \frac{\mu^{2}}{\sqrt{G^{2} N}}, \quad \frac{\lambda_{i}}{\sqrt{G^{2} N} a^{D-2}}, \quad \frac{\tau}{a^{D-3}},
$$

which all have units of mass. For large $L$, the wave function becomes oscillatory. In practice, this forces us to extract the heavy-quark potential from relatively small longitudinal separations: of the order of a few transverse lattice spacings.

\section{APPENDIX B: NONZERO TRANSVERSE MOMENTUM}

In this section, we show how states of nonzero transverse momentum (34) may be handled in the Eguchi-Kawai reduced framework. First, we must choose a phase convention for the overall phase $\overline{\mathbf{x}}$ as a function of $\mathbf{x}_{1}, \mathbf{x}_{2}, \ldots$. We have found a longitudinal momentum weighted center of mass

$$
\overline{\mathbf{x}}=\frac{1}{P^{+}} \sum_{i=1}^{p} k_{i}\left(\mathbf{x}_{i}+\frac{a \hat{\lambda_{i}}}{2}\right)
$$

to be especially convenient. For this choice of $\overline{\mathbf{x}}$, the component $M_{-r}$ of the angular momentum-boost tensor $M_{\mu \nu}$, which generates Lorentz boosts in direction $r$ and obeys

$$
\left[P^{-}, M_{-r}\right]=i P_{r}, \quad\left[P^{+}, M_{-r}\right]=0, \quad\left[P^{r}, M_{-s}\right]=-i g_{s}^{r} P^{+},
$$


takes on a particularly simple form:

$$
\begin{gathered}
e^{-i b^{r} M_{-r}} a_{\lambda}^{\dagger}(k, \mathbf{x}) e^{i b^{r} M}-r=a_{\lambda}^{\dagger}(k, \mathbf{x}) e^{-i k \mathbf{b} \cdot(\mathbf{x}+a \hat{\lambda} / 2)}, \\
e^{-i b^{r} M_{-r}\left|\Psi\left(P^{+}, \mathbf{P}\right)\right\rangle}=\left|\Psi\left(P^{+}, \mathbf{P}-\mathbf{b} P^{+}\right)\right\rangle .
\end{gathered}
$$

In the computation of large- $N$ matrix elements of an operator $\mathcal{O}$,

$$
\begin{aligned}
& \left\langle\Psi^{\prime}\left(P^{+}, \mathbf{P}\right)|\mathcal{O}| \Psi\left(P^{+}, \mathbf{P}\right)\right\rangle \\
& \quad=\left\langle\Psi^{\prime}\left(P^{+}, 0\right)\left|e^{i P^{r} M_{-r} / P^{+}} \mathcal{O} e^{-i P^{r} M_{-r} / P^{+}}\right| \Psi\left(P^{+}, 0\right)\right\rangle,
\end{aligned}
$$

one obtains phase factors in the Eguchi-Kawai reduced theory. These phase factors correspond to any shift in the weighted center of mass (B1). For example, the interaction

$$
\begin{aligned}
\mathcal{O}= & \sum_{\mathbf{x}} \operatorname{Tr}\left\{a_{-\lambda}^{\dagger}\left(k_{1}, \mathbf{x}\right) a_{\lambda}^{\dagger}\left(k_{2}, \mathbf{x}-a \hat{\lambda}\right) a_{\rho}\left(k_{3}, \mathbf{x}-a \hat{\rho}\right)\right. \\
& \left.\times a_{-\rho}\left(k_{4}, \mathbf{x}\right)\right\} \delta\left(k_{1}+k_{2}-k_{3}-k_{4}\right)
\end{aligned}
$$

picks up the phase factor

$$
\begin{aligned}
& e^{i P^{r} M_{-r} / P^{+}} \mathcal{O} e^{-i P^{r} M_{-r} / P^{+}} \\
& \quad=\mathcal{O} \exp \left(-i \frac{a\left(k_{1}+k_{2}\right)}{2 P^{+}} \mathbf{P} \cdot(\hat{\lambda}-\hat{\rho})\right) .
\end{aligned}
$$

We can see from this example that the phase factors are functions only of longitudinal momenta and orientation indices $\lambda$ and $\rho$. Thus Eguchi-Kawai reduction of the theory remains.

Although we have constructed states of definite transverse momentum, the construction of the operator $\mathbf{P}$ itself is somewhat problematic. Consider the most natural definition

$$
\mathbf{P}=\frac{i}{2 a} \sum_{\mathbf{x}, \lambda} \int d k\left[a_{\lambda}^{\dagger}(k, \mathbf{x}+a \hat{r})-a_{\lambda}^{\dagger}(k, \mathbf{x}-a \hat{r})\right] a_{\lambda}(k, \mathbf{x}),
$$

which has the correct classical continuum limit. When we apply this operator to a closed color loop, for example, Eq. (19), we obtain a state that is no longer a closed color loop and whose energy therefore diverges. Evidently, the correct form of $\mathbf{P}$ is quite complicated.

In numerical work, it is advantageous to work with a realvalued representation of the Hamiltonian. We note that, in the $x^{r}$ direction, the boost operator $M_{-r}$ breaks the transverse reflection symmetry $\mathcal{P}^{r}: x^{r} \rightarrow-x^{r}$ of the theory. The boost changes sign under transverse reflections,

$$
\mathcal{P}^{r} e^{i b M_{-r} \mathcal{P}^{r}}=e^{-i b M_{-r}} \quad(\text { no sum over } r) .
$$

For a boost in the $x^{r}$ direction, we introduce a basis with states that are even and odd under $\mathcal{P}^{r}$. We use states of the form

$$
\begin{aligned}
&|\psi A\rangle=\cos \left(\frac{P^{r} M_{-r}}{P^{+}}\right)\left(1+\mathcal{P}^{r}\right)\left|\Psi\left(P^{+}, 0\right)\right\rangle(\text { no sum over } r), \\
&|\psi B\rangle=\sin \left(\frac{P^{r} M_{-r}}{P^{+}}\right)\left(1-\mathcal{P}^{r}\right)\left|\Psi\left(P^{+}, 0\right)\right\rangle
\end{aligned}
$$

$$
\text { (no sum over } r \text { ), }
$$

and the associated phase factors (B7) become sines and cosines. The states $\left|\Psi\left(P^{+}, 0\right)\right\rangle$ are constructed to have definite charge conjugation $\mathcal{C}\left|\Psi\left(P^{+}, 0\right)\right\rangle= \pm\left|\Psi\left(P^{+}, 0\right)\right\rangle$.

\section{APPENDIX C: NUMERICAL METHODS}

To extrapolate to the longitudinal continuum limit, our basic numerical method is DLCQ [19], where we discretize the longitudinal momentum $P^{+}=2 \pi K / \mathcal{L}$ into odd halfintegers in the bound-state equation. $K$ measures the coarseness of the discretization, and $K \rightarrow \infty$ is the longitudinal continuum. This approach has several advantages for numerical work: the resulting Hamiltonian matrix is sparse, and matrix elements of the interaction are easy to compute. The main disadvantage of DLCQ is that numerical convergence tends to be slow. In Appendix $\mathrm{C}$ of $\mathrm{I}$, we introduced an

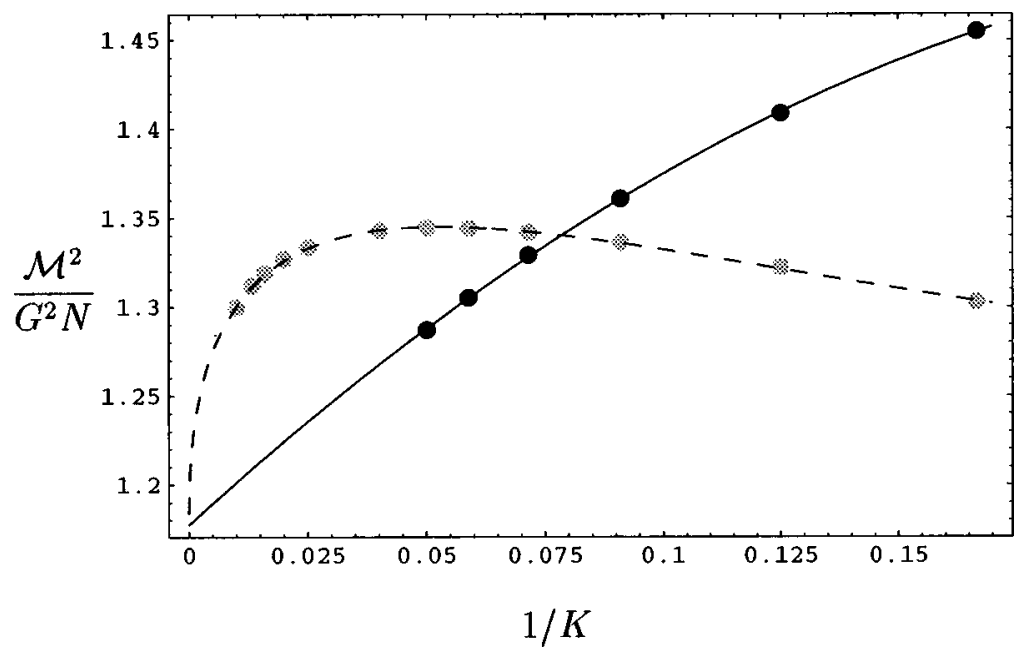

FIG. 13. Convergence of the lowest glueball mass as a function of $1 / K$ for a four-link truncation and $\mu^{2}=\lambda_{i}=0$. The gray points are from conventional DLCQ, and the dashed line is a fit to $1.1834+1.6395 / \sqrt{K}-5.0731 / K+4.3513 / K^{3 / 2}$. The black points are from a calculation using improved matrix elements and are fit to 1.17752 $+2.44169 / K-4.68183 / K^{2}$. 


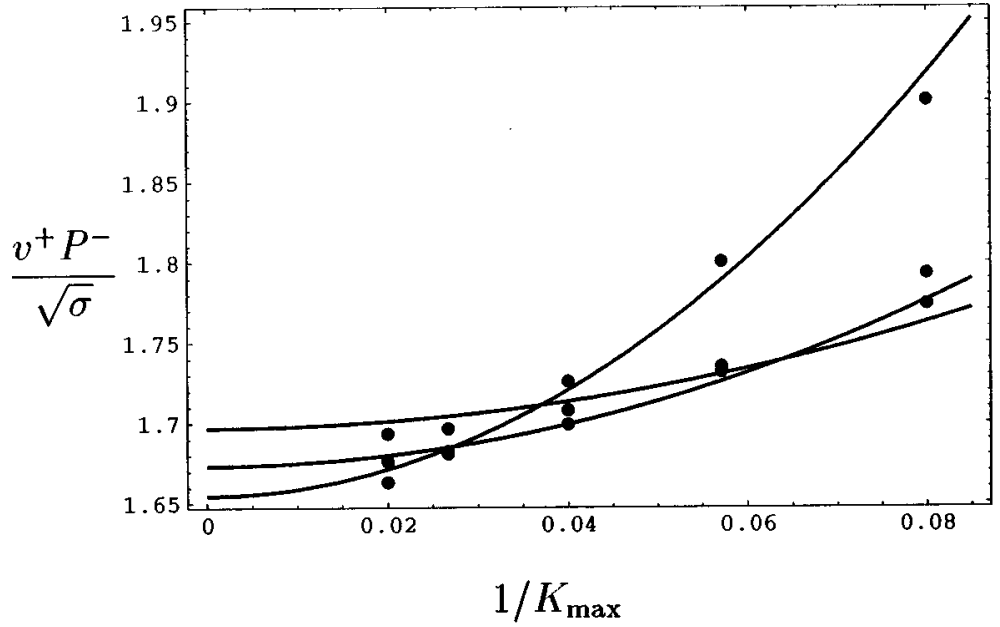

FIG. 14. Convergence of the $|\mathbf{n}|=0$ heavysource potential with $1 / K_{\max }$ for $P_{\max }=4,5,8$ and a two-link truncation. The couplings are from the $m=0.180255$ datum of Table I, and the longitudinal separation is $L=4 / \sqrt{G^{2} N}=1.750 / \sqrt{\sigma}$. The fit function is from Eq. (C3). approach which eliminated the most severe convergence problems, the $1 / K^{2 \beta}$ and $1 / \sqrt{K}$ errors, while minimizing the remaining $1 / K$ error associated with the instantaneous interactions. We have made some subsequent refinements to the method which are described in Appendix D. Because of these improvements, we are able to extrapolate to infinite $K$ with some confidence. For instance, in Fig. 13 we plot the mass eigenvalue of the lowest glueball, calculated using improved matrix elements, as a function of $1 / K$. It is clear that a fit to $c_{0}+c_{1} / K$ should work quite well for data points given in the range $10<K<20$; the improved matrix elements are seen to be necessary for a reliable extrapolation.

In addition to the harmonic resolution $K$, we truncate the Fock space in the number of links $p$. In I we showed that convergence in $p$ is exponentially fast. To estimate the exponent, we calculate the lowest eigenvalue for heavy sources separated by one link, using truncations ( $K, p$-truncation) $=(19 / 2,3),(25 / 2,3),(39 / 2,3),(19 / 2,5),(25 / 2,5),(23 / 2,7)$. Then we fit the eigenvalues to the function

$$
c_{0}+\left(c_{1}+\frac{c_{2}}{K}\right) e^{c_{p} p}+\frac{c_{3}}{K}
$$

to deduce $c_{p}$. It is satisfying that the results for $c_{p}$, typically of order -1 , agree with the analytic estimate from Appendix $\mathrm{B}$ of I. We assume that, for sufficiently large $p$-truncation, $c_{p}$ is a universal constant; it can be equally well applied to the spectra, nonzero winding $|\mathbf{n}|$, and the heavy-source potential.

Using this result for $c_{p}$, we calculate glueball spectra for various $(K, p$-truncation $)=(18 / 2,6) ;(18 / 2,8),(20 / 2,6),(20 /$ $2,8),(24 / 2,6),(32 / 2,6)$ and extrapolate to the continuum using the function

$$
c_{0}+c_{1} e^{c_{p} p}+\frac{c_{2}}{K}
$$

We use the same extrapolation procedure for nonzero winding $|\mathbf{n}| \neq 0$. In this case we use $(|\mathbf{n}|, K, p$-truncation) $=(2,20 / 2,4),(2,20 / 2,6),(2,24 / 2,4),(2,28 / 2,4),(3,21 / 2,5)$, $(3,21 / 2,7),(3,23 / 2,5),(3,27 / 2,5),(4,20 / 2,6),(4,20 / 2,8),(4,22 /$ $2,6),(4,26 / 2,6)$. After extrapolating in $K$ - and $p$-truncation using Eq. (C2), we fit the lowest eigenvalue to the form $\left(\sigma_{T} a|\mathbf{n}|\right)^{2} /\left(G^{2} N\right)+c_{1}+c_{2} /|\mathbf{n}|^{2}$ as discussed in I.

The most tricky calculation is the heavy-source potential. Here we have to extrapolate in $K_{\max }, P_{\max }$, and $p$-truncation. In addition, we must choose reasonable values for the longitudinal separation $L$. For example, in Fig. 14, we see that the eigenvalues are well fit to the function

$$
\frac{v^{+} P^{-}}{\sqrt{\sigma}}=1.64873+\frac{3.08839}{\left(P_{\max }\right)^{3}}+0.64118\left(\frac{P_{\max }}{K_{\max }}\right)^{2},
$$

with a rms deviation of 0.0088 . Although we would expect the leading finite $K_{\max }$ error to be of order $\left(P_{\max } / K_{\max }\right)^{1}$, we find the quadratic term to be much more important. Thus we shall use the fitting function

$$
\frac{v^{+} P^{-}}{\sqrt{G^{2} N}}=c_{0}+\frac{c_{1}}{\left(P_{\max }\right)^{3}}+c_{2}\left(\frac{P_{\max }}{K_{\max }}\right)^{2}+c_{3} e^{c_{p} p} .
$$

In subsequent calculations, we fit to Eq. (C4) using the values

$$
\begin{aligned}
\left(|\mathbf{n}|, K_{\max }, p \text {-truncation, } P_{\max }\right) & \\
= & (0,32 / 2,2,4),(0,32 / 2,4,4),(0,32 / 2,2,5),(0,34 / 2,2,4),(0,44 / 2,2,5),(0,60 / 2,2,4),(1,19 / 2,3,4),(1,19 / 2,5,4), \\
& \times(1,19 / 2,3,5),(1,33 / 2,3,4),(1,33 / 2,3,5),(1,49 / 2,3,4) .
\end{aligned}
$$




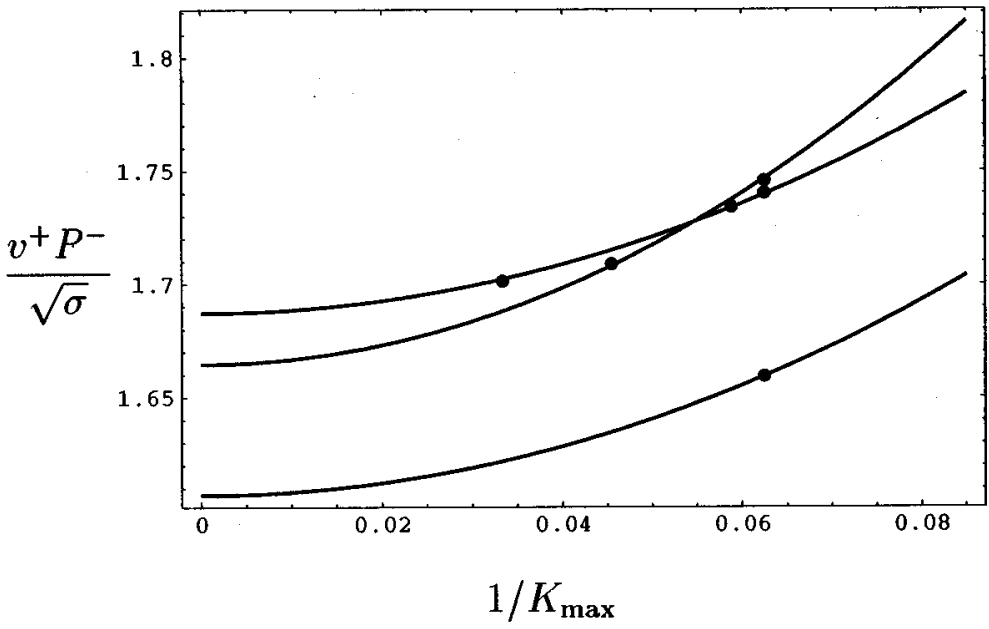

FIG. 15. Convergence of the $|\mathbf{n}|=0$ heavysource potential with $1 / K_{\max }$ for various $P_{\max }$ and $p$-truncations as given by Eq. (C5). The couplings are from the $m=0.180255$ datum of Table I, and $L=4 / \sqrt{G^{2} N}=1.750 / \sqrt{\sigma}$. The fit function is from Eq. (C6).

Note that for the heavy-source calculation, the conserved quantity $\mathbf{n}$, analogous to winding number, is the number of links minus number of antilinks, that is, the transverse separation of the sources in lattice units. Example data points are shown in Fig. 15 along with the associated fit function

$$
\begin{aligned}
\frac{v^{+} P^{-}}{\sqrt{\sigma}}= & 1.54997+\frac{2.95576}{\left(P_{\max }\right)^{3}}+0.838171\left(\frac{P_{\max }}{K_{\max }}\right)^{2} \\
& +0.773708 e^{c_{p} p}
\end{aligned}
$$

In order to measure the heavy-source potential and optimize its rotational invariance, we calculate the potential for $|\mathbf{n}|$ $=0$ and $L \sqrt{G^{2} N}=3,4$, and 6 . Then we fit to the functional form [27]

$$
\frac{v^{+} P^{-}}{\sqrt{G^{2} N}}=c_{0} L \sqrt{G^{2} N}+c_{1}+\frac{c_{2}}{L \sqrt{G^{2} N}} .
$$

Next, we calculate the potential for $|\mathbf{n}|=1$ and $L \sqrt{G^{2} N}$ $=3$; we then demand rotational invariance for this datum.

\section{APPENDIX D: IMPROVED MATRIX ELEMENTS FOR DLCQ}

In this section, we will describe some refinements to the basic technique introduced in Appendix C of I. Our method is the following: when calculating the matrix elements of the Hamiltonian in a many-particle calculation, we will use ordinary DLCQ commutation relations to calculate contractions associated with spectator particles. To calculate the interaction itself, we take the DLCQ momenta of the interacting particles, project onto a smooth wave function basis (continuous longitudinal momentum), and calculate matrix elements of the interaction in this smooth wave function basis. Our modification of DLCQ essentially eliminates the $1 / \sqrt{K}$ and $1 / K^{2 \beta}$ errors associated with small momenta. The remaining errors include a (small) $1 / K$ error from the instantaneous interaction $1 /\left(k^{+}-p^{+}\right)^{2}, 1 / K^{2}$ errors from DLCQ itself, and a $1 / K$ error from production of particles at small momentum. In the following, we discuss improvements to the $\quad(2$ particles $) \rightarrow(2$ particles $) \quad$ interactions, the (1 particle $) \rightarrow(3$ particles $)$ interactions, and the heavy source interactions. The "particles" in the present context are the link fields of course.

\section{1. ( 2 particles $) \rightarrow(2$ particles $)$ interactions}

Consider a typical four-point interaction

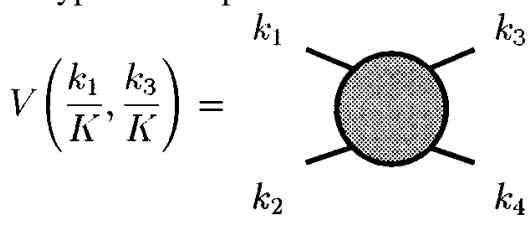

(D1)

with DLCQ momenta $k_{i} \in\{1 / 2,3 / 2, \ldots\}$ and $K=k_{1}+k_{2}=k_{3}$ $+k_{4}$. In particular, the mass term is treated as if it were a four-particle interaction:

$$
V(x, y)=\frac{\mu^{2}}{2}\left(\frac{1}{x}+\frac{1}{1-x}\right) \delta(x-y) .
$$

We start by calculating eigenfunctions of the two-particle bound-state equation [2]:

$$
\begin{aligned}
\mathcal{M}^{2} f(x, 1-x)= & \int_{0}^{1} d y H(x, y) f(y, 1-y) \\
= & \frac{\mu^{2} f(x, 1-x)}{x(1-x)} \\
& -\frac{G^{2} N}{4 \pi} \int_{0}^{1} d y \frac{f(y, 1-y)}{\sqrt{x(1-x) y(1-y)}} \\
& \times\left(\frac{(x+y)(2-x-y)}{(x-y)^{2}}+1\right),
\end{aligned}
$$

where $\lambda_{1}+\lambda_{2} / 2+\lambda_{3}=-G^{2} N a^{D-2}$. Then we write the incoming and outgoing DLCQ states as linear combinations of 
the first $K$ eigenfunctions and evaluate matrix elements of the various interactions $V(x, y)$ in the eigenfunction basis.

We introduce a basis of even and odd wave functions (labeled by subscripts $s$ and $s^{\prime}$ ):

$$
\begin{aligned}
\phi_{s}(x)= & {[x(1} \\
& -x)]^{\beta} \begin{cases}\cos \left[\pi s\left(x-\frac{1}{2}\right)\right], & s \in\{0,2, \ldots, 2 S\}, \\
\sin \left[\pi s\left(x-\frac{1}{2}\right)\right], & s \in\{1,3, \ldots, 2 S-1\},\end{cases}
\end{aligned}
$$

with $\beta$ given by $\mu^{2}=G^{2} N \beta \tan (\pi \beta), 0<\beta<1 / 2$. We define, for both even and odd wave functions, matrix elements of the various interaction terms $V_{s, s^{\prime}}$ $=\int d x d y \phi_{s}(x) V(x, y) \phi_{s^{\prime}}(y)$, the inner product matrix $E_{s, s^{\prime}}=\int d x d y \phi_{s}(x) \phi_{s^{\prime}}(y)$, and the two-particle Hamiltonian $H_{s, s^{\prime}}=\int d x d y \phi_{s}(x) H(x, y) \phi_{s^{\prime}}(y)$. In addition, we define the matrix $T_{i, s}$ which maps the wave function basis onto the DLCQ basis:

$$
T_{i, s}=\left(\frac{k_{1} k_{2}}{K^{2}}\right)^{\beta}\left\{\begin{array}{c}
\cos \left[\pi s\left(\frac{k_{1}-k_{2}}{2 K}\right)\right], \\
\sin \left[\pi s\left(\frac{k_{1}-k_{2}}{2 K}\right)\right],
\end{array}\right.
$$

where the subscript $i \in\{1,2, \ldots K\}$ denotes a two-particle DLCQ basis state with momenta $k_{1}=i-1 / 2, k_{2}=K-k_{1}$.

This choice of basis wave functions has several advantages over our previous choice. First, this basis is numerically "well conditioned," while the previous choice was numerically unstable. Second, the highly excited eigenfunctions of Eq. (D3) are themselves nearly sines and cosines. We now generate the improved matrix elements at the beginning of a calculation, store them in memory, and use them as needed.

One calculates the various integrals using the formula

$$
\begin{gathered}
\int_{0}^{1} d x x^{\mu}(1-x)^{\mu} \cos [2 a(x-1 / 2)] \\
=\sqrt{\pi} \Gamma(\mu+1) f_{\mu+1 / 2}(2 a),
\end{gathered}
$$

where $f_{\mu}(x)$ is defined in terms of a Bessel function:

$$
\begin{aligned}
f_{\mu}(z) & =J_{\mu}(z / 2) / z^{\mu}, \\
\frac{d}{d x} f_{\mu}(x) & =-\frac{x}{2} f_{\mu+1}(x), \\
4 \mu f_{\mu}(x) & =f_{\mu-1}(x)+x^{2} f_{\mu+1}(x), \\
\lim _{x \rightarrow 0} f_{\mu}(x) & =\frac{1}{\Gamma(\mu+1) 2^{2 \mu}} .
\end{aligned}
$$

Thus, for example,

$$
\begin{aligned}
E_{s, s^{\prime}}= & \frac{\sqrt{\pi}}{2} \Gamma(2 \beta+1)\left[f_{2 \beta+1 / 2}\left(\pi\left(s-s^{\prime}\right)\right)\right. \\
& \left. \pm f_{2 \beta+1 / 2}\left(\pi\left(s+s^{\prime}\right)\right)\right] .
\end{aligned}
$$

The greatest difficulty comes from the instantaneous interaction

$$
V_{s, s^{\prime}}=-\frac{G^{2} N}{8 \pi} \int d x d y \frac{(x+y)(2-x-y) \phi_{s}(x) \phi_{s^{\prime}}(y)}{\sqrt{x y(1-x)(1-y)}(x-y)^{2}} .
$$

We cannot solve this integral analytically, but we can subtract the pole part at $x=y$ and solve the remaining finite integral numerically. Thus we add and subtract the quantity

$$
\begin{aligned}
& -\frac{G^{2} N}{8 \pi} \int d x d y \frac{(x+y)(2-x-y)[x(1-x) y(1-y)]^{\beta-1 / 2}}{(x-y)^{2}} \\
& \quad \times\left[\frac{1}{2} \cos \left(\frac{\pi}{2}\left(s-s^{\prime}\right)(x+y-1)\right)\right. \\
& \left.\quad \pm \frac{1}{2} \cos \left(\frac{\pi}{2}\left(s+s^{\prime}\right)(x+y-1)\right)\right]
\end{aligned}
$$

which, using the identity

$$
\frac{1}{(x-y)^{2}}=-\lim _{\epsilon \rightarrow 0} \int_{0}^{\infty} d z z \cos [z(x-y)] e^{-\epsilon z},
$$

equals

$$
\begin{aligned}
G^{2} N & \frac{\Gamma(\beta+1 / 2)}{16} \\
& \times\left\{f_{\beta}\left(v^{-}\right)\left[(1+4 \beta) f_{\beta}\left(v^{-}\right)-2\left(v^{-}\right)^{2} f_{\beta+1}\left(v^{-}\right)\right]\right. \\
& \left. \pm f_{\beta}\left(v^{+}\right)\left[(1+4 \beta) f_{\beta}\left(v^{+}\right)-2\left(v^{+}\right)^{2} f_{\beta+1}\left(v^{+}\right)\right]\right\},
\end{aligned}
$$

where $v^{ \pm}=\pi\left(s \pm s^{\prime}\right) / 2$. The numerical integration of Eq. (D12) minus Eq. (D13) is performed using the Gauss-Jacobi method [30], which correctly handles the end-point behavior of the integrand and converges exponentially fast.

We solve the generalized eigenvalue problem $H \chi_{t}$ $=h_{t} E \chi_{t}$, where the eigenvectors $\chi_{t}$ have normalization $\chi_{t^{\prime}}^{\dagger} E \chi_{t}=\delta_{t, t^{\prime}}$. Using the lowest $K$ eigenvectors, we define an eigenstate basis (denoted by subscripts $t$ and $t^{\prime}$ ):

$$
T_{i, t}=T_{i, s}\left(\chi_{t}\right)_{s}
$$

To make the transformation between the DLCQ and eigenfunction bases orthonormal, we perform a standard (QR) factorization [30], $T_{i, t}=Q_{i, t^{\prime}} R_{t^{\prime}, t}$, and discard the triangular matrix $R$ (diagonal elements of $R$ must be positive). Finally, we define the improved matrix elements by

$$
V_{i, j}=Q_{i, t}\left(\chi_{t}\right)_{s}^{\dagger} V_{s, s^{\prime}}\left(\chi_{t^{\prime}}\right)_{s^{\prime}} Q_{t^{\prime}, j}^{T}
$$



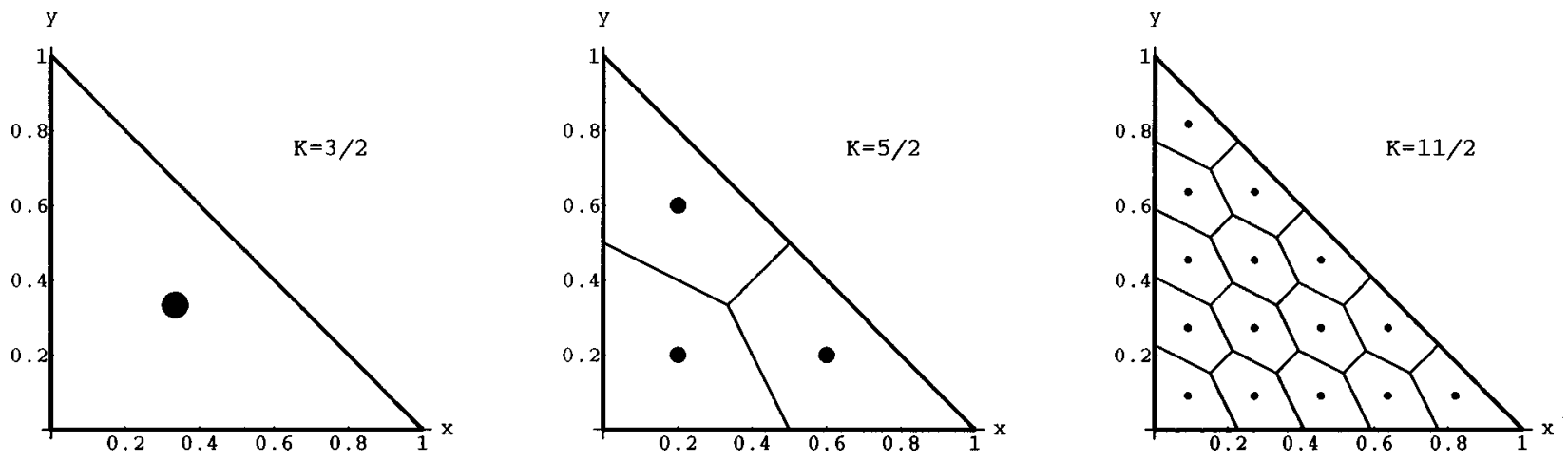

FIG. 16. Region $R_{k_{1}, k_{2}, k_{3}}$ for $K=3 / 2, K=5 / 2$, and $K=11 / 2$. The dots represent the points $\left(k_{1} / K, k_{2} / K\right)$, and $R_{k_{1}, k_{2}, k_{3}}$ is the surrounding polygonal region.

\section{2. (1 particle $) \rightarrow(3$ particles $)$ interactions}

From the general bound-state equations of twodimensional gauge theories with pair production, one can show that as the momenta of any two particles go to zero the wave function becomes constant [29]. Thus we define a basis of wave functions

$$
\phi_{k_{1}, k_{2}, k_{3}}(x, y)=C_{k_{1}, k_{2}, k_{3}}\left\{\begin{array}{c}
\frac{[x y(1-x-y)]^{\beta}}{[(x+y)(1-x)(1-y)]^{2 \beta}}, \\
(x, y) \in R_{k_{1}, k_{2}, k_{3},}, \\
0, \quad \text { otherwise, }
\end{array}\right.
$$

with normalization

$$
1=\int_{0}^{1} d x \int_{0}^{1-x} d y \phi_{k_{1}, k_{2}, k_{3}}(x, y)^{2}
$$

for three outgoing particles with longitudinal momentum fractions $x, y$, and $1-x-y$ in the continuous wave function basis and $k_{i} \in\{1 / 2,3 / 2, \ldots\}, K=k_{1}+k_{2}+k_{3}$, in the DLCQ basis. We define the polygonal region $R_{k_{1}, k_{2}, k_{3}}$ surrounding $\left(k_{1} / K, k_{2} / K\right)$ as shown in Fig. 16.

Consider a typical four-point interaction

$$
V\left(\frac{k_{1}}{K}, \frac{k_{2}}{K}, \frac{k_{3}}{K}\right)=K \longrightarrow{ }_{k_{2}}^{k_{1}} .
$$

We calculate improved matrix elements associated with interaction $V(x, y, 1-x-y)$ using the expression

$$
V_{k_{1}, k_{2}, k_{3}}=\int_{0}^{1} d x \int_{0}^{1-x} d y \phi_{k_{1}, k_{2}, k_{3}}(x, y) V(x, y, 1-x-y) \text {. }
$$

The integrals are calculated numerically, paying careful attention to the small momentum regions.

\section{Heavy-source interactions}

Improvement of the matrix elements for the interactions between the heavy sources and the link fields is particularly challenging because of the oscillatory nature of the wave function for large separation $L$ and the presence of the cutoff $P_{\max }$. So far, we have concentrated on removing the leading errors which are, as before, associated with small longitudinal momenta and are of order $1 /\left(K_{\max }\right)^{2 \beta}$ and $1 / \sqrt{K_{\max }}$. At this point, it makes no sense to include the finite- $L$ phase factors in the improvement scheme because we will also neglect oscillation of the wave function itself in the improved matrix elements. The leading error associated with the improved matrix elements is of order $1 / K_{\max }$.

For (source-link $) \rightarrow($ source-link) interactions, we define wave functions

$$
\phi_{k}(x)=C_{k} \begin{cases}x^{\beta}, & k-1 / 2<x<k+1 / 2 \\ 0, & \text { otherwise }\end{cases}
$$

where $k, k^{\prime} \in\{1 / 2,3 / 2, \ldots\}$, restricted by $K_{\text {max }} \geqslant K_{\text {link }}$, is the DLCQ momentum of the link field neighboring the heavy source and $x$ is its continuum longitudinal momentum rescaled by $K_{\max } /\left(v^{+} \sqrt{G^{2} N} P_{\max }\right)$. The normalization is 1 $=\int d x \phi_{k}(x)^{2}$. We calculate improved matrix elements for the various interactions using

$$
V_{k, k^{\prime}}=\int d x d y \phi_{k}(x) V(x, y) \phi_{k^{\prime}}(y),
$$

with the finite- $L$ phase factor replaced by its DLCQ value

$$
\exp \left(\frac{i L \sqrt{G^{2} N}(x-y) P_{\max }}{2 K_{\max }}\right) \rightarrow \exp \left(\frac{i L \sqrt{G^{2} N}\left(k-k^{\prime}\right) P_{\max }}{2 K_{\max }}\right) .
$$

In the case of the mass term for the link field adjacent to the heavy source, we assign half of the contribution to the link-link interactions acting on the interior of the string, consistent with Eq. (D2), and the other half to the heavy-link interaction. Thus we use 


$$
V(x, y)=\frac{\mu^{2} K_{\max }}{2 x \sqrt{G^{2} N} P_{\max }} \delta(x-y) .
$$

For the instantaneous $A_{+}$exchange interaction, we must be careful about how we handle the $1 /(x-y)^{2}$ pole and the $K_{\max } \geqslant K_{\text {link }}$ cutoff. For the off-diagonal part, we use

$$
\begin{aligned}
V_{k, k^{\prime}}= & -\frac{\sqrt{G^{2} N} K_{\max }}{8 \pi\left(k-k^{\prime}\right)^{2} P_{\max }} \exp \left(\frac{i L \sqrt{G^{2} N}\left(k-k^{\prime}\right) P_{\max }}{2 K_{\max }}\right) \\
& \times \int d x d y \frac{x+y}{\sqrt{x y}} \phi_{k}(x) \phi_{k^{\prime}}(y), \quad k \neq k^{\prime} .
\end{aligned}
$$

If the $1 /(x-y)^{2}$ pole were left inside the integral, there would be a logarithmic divergence when $k=k^{\prime} \pm 1$. The diagonal part is obtained by adding and subtracting the "selfinertia"

$$
\begin{aligned}
V_{k, k}= & \sum_{k^{\prime} \neq k}^{K_{\max } \geqslant K_{\text {link }}}\left|V_{k, k^{\prime}}\right|\left(\frac{k^{\prime}}{k}\right)^{\beta} \\
& -\frac{\sqrt{G^{2} N} K_{\max }}{8 \pi P_{\max }} \int_{0}^{K^{\prime}} d y \frac{k+y}{\sqrt{k y}(k-y)^{2}}\left(\frac{y}{k}\right)^{\beta},
\end{aligned}
$$

where $K^{\prime}=K_{\max }-K_{\text {link }}+k$. The integral is solved numerically with an implicit principle value prescription for the pole at $y=k$. This method for handling the self-inertias is discussed in Ref. [31].

Turning our attention to the (source) $\rightarrow$ (source +2 links) interactions, we proceed in an analogous manner. Since the wave function becomes constant as any two momenta go to zero, we define the wave functions

$$
\phi_{k_{1}, k_{2}}(x, y)=C_{k_{1}, k_{2}}\left\{\begin{array}{l}
\frac{(x y)^{\beta}}{(x+y)^{2 \beta}}, \quad k_{1}-1 / 2<x<k_{1}+1 / 2 \text { and } k_{2}-1 / 2<y<k_{2}+1 / 2 \\
0, \quad \text { otherwise, }
\end{array}\right.
$$

where $k_{1}, k_{2} \in\{1 / 2,3 / 2, \ldots\}$ are the DLCQ momenta of the two outgoing link fields created adjacent to the source. The normalization is $1=\int d x d y \phi_{k_{1}, k_{2}}(x, y)^{2}$. We calculate improved matrix elements for the various interactions using

$$
V_{k_{1}, k_{2}}=\int d x d y \phi_{k_{1}, k_{2}}(x, y) V(x, y) .
$$

As before, the finite $L$ phase factors in $V(x, y)$ are replaced by their DLCQ values.

[1] S. Dalley and B. van de Sande, Nucl. Phys. B (Proc. Suppl.) 53, 827 (1997); Phys. Rev. D 56, 7917 (1997).

[2] W. A. Bardeen and R. B. Pearson, Phys. Rev. D 14, 547 (1976).

[3] W. A. Bardeen, R. B. Pearson, and E. Rabinovici, Phys. Rev. D 21, 1037 (1980).

[4] G. Mack, Nucl. Phys. B235, 197 (1984); H. B. Nielsen and A. Patkos ibid. B195, 137 (1982); H.-J. Pirner, Prog. Part. Nucl. Phys. 29, 33 (1992).

[5] S. J. Brodsky, presented at the NuSS, Korea, 1997, Report No. SLAC-PUB-7645, hep-ph/9710288.

[6] G. 't Hooft, Nucl. Phys. B72, 461 (1974); B75, 461 (1974).

[7] M. Burkardt, Phys. Rev. D 54, 2913 (1996).

[8] M. Teper, Phys. Rev. D 59, 014512 (1999).

[9] S. Dalley and T. R. Morris, Int. J. Mod. Phys. A 5, 3929 (1990).

[10] M. Burkardt and B. Klindworth, Phys. Rev. D 55, 1001 (1997).

[11] K. G. Wilson, Phys. Rev. D 10, 2445 (1974).

[12] P. A. Griffin, Nucl. Phys. B139, 270 (1992).

[13] D. Weingarten, Phys. Lett. 90B, 220 (1980).

[14] K. G. Wilson, in Recent Developments of Gauge Theories, edited by G. 't Hooft et al. (Plenum, New York, 1980).

[15] J. B. Kogut and L. Susskind, Phys. Rev. D 9, 3501 (1974).

[16] H.-J. Pirner, J. Wroldsen, and M. Ilgenfritz, Nucl. Phys. B294, 905 (1987); B. Grossman et al., Int. J. Mod. Phys. A 6, 2649 (1991).

[17] V. A. Franke, S. A. Paston, and E. V. Prokhvatilov, Report No. SPbU-IP-98-5, hep-th/9803035.

[18] A. Casher, Phys. Rev. D 14, 452 (1976); T. Maskawa and K. Yamawaki, Prog. Theor. Phys. 56, 270 (1976); C. Thorn, Phys. Lett. 70B, 77 (1977).

[19] H.-C. Pauli and S. J. Brodsky, Phys. Rev. D 32, 1993 (1985); 32, 2001 (1985).

[20] S. Dalley and I. R. Klebanov, Phys. Rev. D 47, 2517 (1993); K. Demeterfi, I. R. Klebanov, and G. Bhanot, Nucl. Phys. B418, 15 (1994); F. Antonuccio and S. Dalley, ibid. B461, 275 (1996); Phys. Lett. B 376, 154 (1996).

[21] J. B. Kogut and L. Susskind, Phys. Rev. D 11, 395 (1975).

[22] D. A. Soper, Nucl. Phys. B (Proc. Suppl.) 53, 69 (1997).

[23] T. Eguchi and H. Kawai, Phys. Rev. Lett. 48, 1063 (1983).

[24] G. Bhanot, U. M. Heller, and H. Neuberger, Phys. Lett. 113B, 47 (1982).

[25] C. B. Thorn, Phys. Rev. D 19, 639 (1979); 20, 1435 (1979). 
[26] E. Witten, in Recent Developments of Gauge Theories [14].

[27] M. Lüscher, K. Symanzik, and P. Weisz, Nucl. Phys. B173, 365 (1980).

[28] Q. Z. Chen, X. Q. Luo, S. H. Guo, and X. Y. Fang, Phys. Lett. B 348, 560 (1995).

[29] S. Dalley, Phys. Rev. D 58, 087705 (1998).
[30] W. H. Press, B. P. Flannery, S. A. Teukolsky, and W. T. Vetterling, Numerical Recipes (Cambridge University Press, Cambridge, England, 1989); G. H. Golub and C. F. Van Loan, Matrix Computations (Johns Hopkins University Press, Baltimore, 1983).

[31] B. van de Sande, Phys. Rev. D 54, 6347 (1996). 